ARTICLE

\title{
Structural basis for extracellular cis and trans RPTP $\sigma$ signal competition in synaptogenesis
}

Charlotte H. Coles ${ }^{1, \dagger}$, Nikolaos Mitakidis ${ }^{1}$, Peng Zhang ${ }^{2}$, Jonathan Elegheert ${ }^{1}$, Weixian Lu', Andrew W. Stoker ${ }^{3}$, Terunaga Nakagawa ${ }^{4}$, Ann Marie Craig ${ }^{2}$, E. Yvonne Jones ${ }^{1} \&$ A. Radu Aricescu ${ }^{1}$

Receptor protein tyrosine phosphatase sigma (RPTP $\sigma$ ) regulates neuronal extension and acts as a presynaptic nexus for multiple protein and proteoglycan interactions during synaptogenesis. Unknown mechanisms govern the shift in RPTP $\sigma$ function, from outgrowth promotion to synaptic organization. Here, we report crystallographic, electron microscopic and small-angle $\mathrm{X}$-ray scattering analyses, which reveal sufficient inter-domain flexibility in the RPTP $\sigma$ extracellular region for interaction with both cis (same cell) and trans (opposite cell) ligands. Crystal structures of RPTP $\sigma$ bound to its postsynaptic ligand TrkC detail an interaction surface partially overlapping the glycosaminoglycan-binding site. Accordingly, heparan sulphate and heparin oligomers compete with TrkC for RPTP $\sigma$ binding in vitro and disrupt TrkC-dependent synaptic differentiation in neuronal co-culture assays. We propose that transient RPTP $\sigma$ ectodomain emergence from the presynaptic proteoglycan layer allows capture by TrkC to form a trans-synaptic complex, the consequent reduction in RPTP $\sigma$ flexibility potentiating interactions with additional ligands to orchestrate excitatory synapse formation.

\footnotetext{
${ }^{1}$ Division of Structural Biology, Wellcome Trust Centre for Human Genetics, University of Oxford, Roosevelt Drive, Oxford OX3 7BN, UK. ${ }^{2}$ Brain Research Centre and Department of Psychiatry, University of British Columbia, Vancouver, British Columbia, Canada V6T 2B5. ${ }^{3}$ Cancer Section, Institute of Child Health, University College London, 30 Guilford Street, London WC1N 1EH, UK. ${ }^{4}$ Department of Molecular Physiology and Biophysics, Vanderbilt University, School of Medicine, 702 Light Hall (0615), Nashville, Tennessee 37232-0615, USA. † Present address: Laboratory for Axon Growth and Regeneration, German Center for Neurodegenerative Diseases (DZNE), Ludwig-Erhard-Allee 2, 53175 Bonn, Germany. Correspondence and requests for materials should be addressed to A.R.A. (email: radu@strubi.ox.ac.uk) or to E.Y.J. (email: yvonne@strubi.ox.ac.uk)
} 
$\mathrm{N}$ euronal synaptogenesis is orchestrated by cell surface receptors, which define the formation and functionality of distinct synapse classes ${ }^{1,2}$. These 'organizer' molecules can act as scaffolds or hubs, to integrate multiple inputs into a unified cellular response. This concept is well established for intracellular systems, but only now emerging for cell surface molecules such as neurexin-neuroligin and repulsive guidance molecule (RGM)-neogenin protein complexes ${ }^{3-5}$. At the axonal surface, type IIa receptor protein tyrosine phosphatases (RPTPs) (type IIa RPTPs, for example, RPTP $\sigma$, RPTP $\delta$ and leukocyte common antigen-related (LAR) in vertebrates, dLAR in Drosophila), are a recently identified nexus for extracellular interactions, receiving signals and transmitting them intracellularly to the cytoskeleton to regulate neuronal extension and guidance, as well as synaptic organization ${ }^{6-9}$.

Heparan sulphate proteoglycans (HSPGs) and chondroitin sulphate proteoglycans (CSPGs) play important roles in the modulation of RPTP signalling in the nervous system ${ }^{10-15}$. At the Drosophila neuromuscular junction, the HSPGs dSyndecan and dDallylike opposingly regulate dLAR-mediated synaptic morphogenesis and active zone function ${ }^{12}$. While the interaction of dLAR with presynaptic dSyndecan promotes bouton growth, postsynaptic dDallylike competes with dSyndecan for dLAR binding, leading to an inhibition of growth and active zone stabilization $^{12}$. Interactions of the RPTP $\sigma$ ectodomain with HSPGs and CSPGs modulate axonal growth both during development and post injury ${ }^{10,13,14,16}$. HSPGs cluster RPTP $\sigma$, a characteristic proposed to drive a localized imbalance of protein tyrosine phosphorylation and hence promote growth ${ }^{14}$. Once the axon has reached its final target, RPTP $\sigma$ can establish direct interactions with multiple postsynaptic proteins. In vertebrates, these include the TrkC receptor protein tyrosine kinase, Netrin-G ligand-3 (NGL-3), interleukin-1 receptor accessory protein and Slit- and Trk-like receptors 1 and 2 (Slitrk1 and Slitrk2) ${ }^{17-20}$ These trans-synaptic complexes mediate bi-directional excitatory synapse formation, simultaneously triggering presynaptic differentiation and an accumulation of synaptic vesicles, and clustering of the postsynaptic density 7,9 .

There are two major neuronal RPTP $\sigma$ isoforms, sharing a common intracellular catalytic region and an extracellular region predicted to contain three immunoglobulin (Ig)-like domains followed by either five or nine fibronectin (FN) type III domains, in the central and peripheral nervous systems, respectively ${ }^{21}$. Further isoforms include a combination of four mini-exons (meA-meD) that may modulate interactions with protein partners (Fig. 1a) ${ }^{8,21}$. Previous mutagenesis and structural studies have demonstrated that the proteoglycan-binding site lies on Ig1 of RPTP $\sigma$, and comprises an extended positively charged surface of basic residues ${ }^{10,13,14}$. Binding of postsynaptic TrkC, is reported to require the N-terminal three Ig domains of $\mathrm{RPTP}^{17}$; the NGL-3-binding site has been mapped to the FN1-2 domains $^{18}$.

The properties that fit the RPTP $\sigma$ ectodomain for its function as an integrative hub for signalling in synaptogenesis are unknown. Here we report a molecular level analysis of the RPTP $\sigma$ ectodomain and of its direct interactions with the postsynaptic binding partner TrkC. We reveal that the multidomain extracellular region of RPTP $\sigma$ is unexpectedly flexible. This characteristic confers sufficient conformational freedom to allow its binding to both pre- or postsynaptic ligands. RPTPo:TrkC crystal structures provide an explanation for the specificity of this interaction and also highlight an overlap of TrkC and proteoglycan-binding sites on RPTP $\sigma$. This observation suggests that there is competition between $\mathrm{TrkC}$ and heparan a

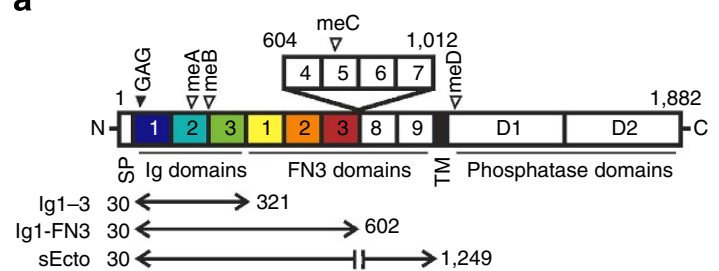

b

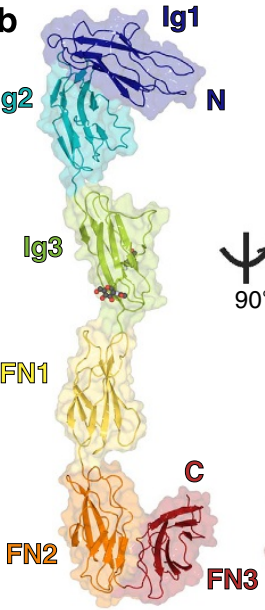

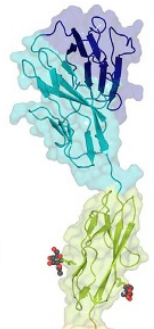

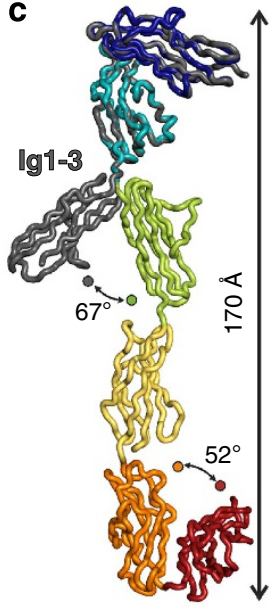

d

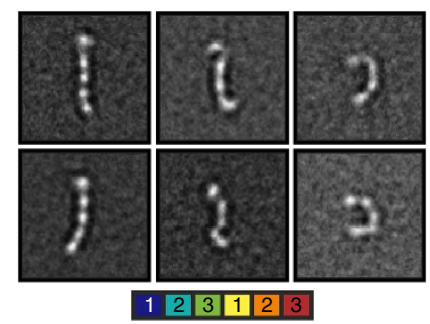

e

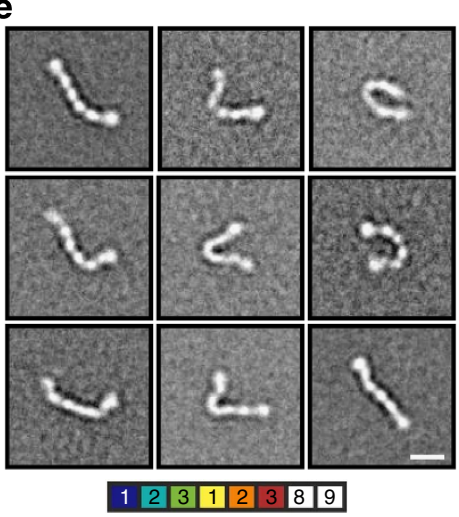

Figure 1 | RPTP $\boldsymbol{\sigma}$ ectodomain flexibility. (a) RPTP $\sigma$ domain organization. $\mathrm{N}$, amino-terminus (extracellular); SP, secretion signal peptide; TM, transmembrane; C, C terminus (intracellular); Ig, immunoglobulin-like; FN, fibronectin type-III; GAG, glycosaminoglycan-binding site (filled arrowhead). Alternative splicing inserts: FN domains 4-7 and mini-exons A-D (open arrowhead). (b) Ribbon and surface representations of the human RPTP $\sigma$ Ig1-FN3 crystal structure. $\mathrm{N}$-linked glycans in atom representation. (c) lg3 movement in Ig1-FN3 relative to Ig1-3 (grey, PDB ID: 2YD9) structure.

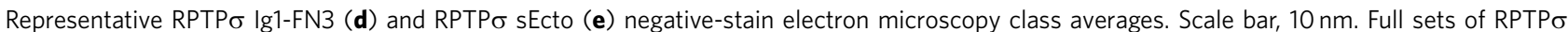
Ig1-FN3 and sEcto class averages are provided in Supplementary Figs 2 and 3. 
sulphate for RPTP $\sigma$ binding, and we provide support for this notion in biophysical and cellular assays. Overall, our study provides novel insights into the mechanisms determining the hierarchy and functional consequences of RPTP $\sigma$-ligand interactions.

\section{Results}

The RPTPo ectodomain exhibits extensive flexibility. We first investigated the molecular characteristics of RPTP $\sigma$ that allow presentation of the N-terminal Ig domains for both pre- and postsynaptic ligand binding. We determined the $3.15 \AA$ crystal structure of a six N-terminal domain human RPTP $\sigma$ construct (termed Ig1-FN3, Fig. 1a), which contains all the binding sites for synaptic ligands identified to date. This construct maintains the V-shaped Ig1-2 arrangement previously reported ${ }^{14,22}$, followed by an extended conformation of the sequential domains Ig3, FN1 and FN2 (Fig. 1b; Table 1). Superposition of Ig1-FN3 with the crystal structure of Ig1-3 (ref. 14) revealed a hinge point between domains Ig2 and Ig3 (Fig. 1c). The four amino-acid meB exon would extend this apparently flexible linker further, by four residues (Supplementary Fig. 1). While FN1 and FN2 domains align approximately with the long axis of the molecule, the C-terminal FN3 domain folds back, suggesting the FN2-3 linker may also be a flexion point. Since no substantial Ig3-FN1, FN1-FN2 or FN2-FN3 inter-domain interfaces are apparent, we hypothesized that when released from crystal packing constraints each of the Ig2-FN3 region linkers may provide substantial flexibility. To test this, single-particle negative-stain electron microscopy (EM) class averages of human RPTP $\sigma$ Ig1-FN3 were calculated. These showed a broad range of conformations (Fig. 1d; Supplementary Fig. 2). This flexibility, reminiscent of hinge points in $\alpha$ neurexin ${ }^{23}$, is in marked contrast to the rigidity of homophilic cell adhesion molecules of similar size and domain organization, such as cadherins, RPTP $\mu$ and SYG-1/SYG-2 (refs 24-26).

We extended our EM analysis to the full ectodomain of the eight-domain isoform of human RPTP $\sigma$ (sEcto; Fig. 1a,e; Supplementary Fig. 3). The 150 class averages generated reinforce our conclusions from the six-domain structural analyses. The RPTP $\sigma$ ectodomain exhibits a surprisingly large flexibility, with observed conformations ranging from almost fully extended, to essentially bent double (Fig. 1e; Supplementary Fig. 3). To control for the potential risk of artefacts associated with negative staining, we also performed small-angle X-ray scattering (SAXS) measurements, at a physiological $\mathrm{pH}$ (7.4), for both human RPTP $\sigma$ Ig1FN3 and sEcto. This analysis further supports the observation that both proteins are flexible and are likely to adopt multiple conformations in solution (Supplementary Fig. 4). Taken together, crystallographic, EM and SAXS analyses demonstrate that the ectodomain of RPTP $\sigma$ is able to explore a large conformational space.

Structural analysis of the RPTPo:TrkC trans-synaptic complex. How do these conformational properties contribute to the interaction of RPTP $\sigma$ with ligands? We sought to compare and

Table 1 | Data collection and refinement statistics.

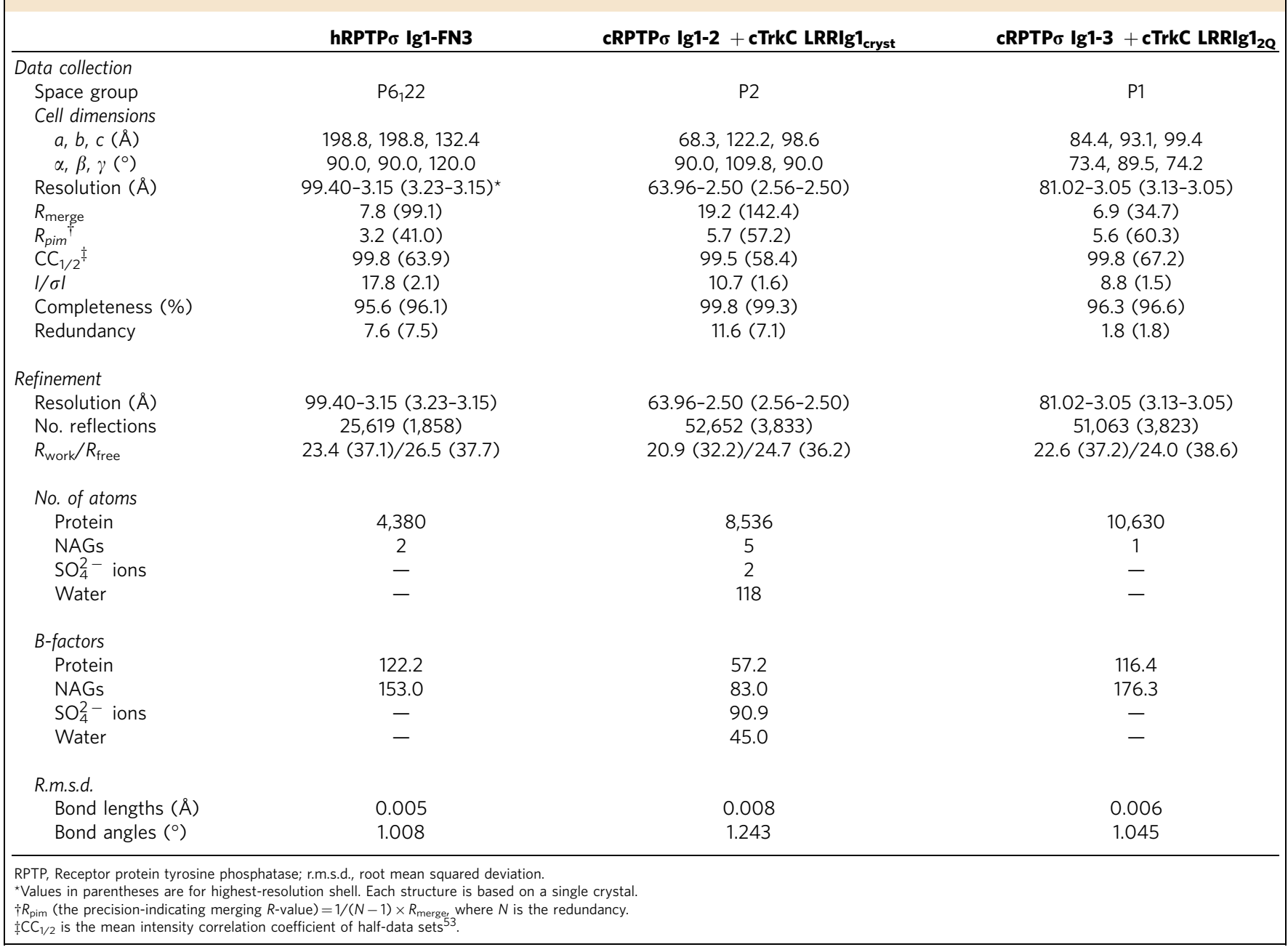


contrast complex formation between the RPTP $\sigma$ N-terminal region and axonal HSPGs ${ }^{14}$ with the trans-synaptic interaction between RPTP $\sigma$ and TrkC. The TrkC ectodomain comprises an $\mathrm{N}$-terminal leucine-rich repeat (LRR) domain, followed by two Ig domains (Fig. 2a). Equilibrium surface plasmon resonance (SPR) assays confirmed previously reported data ${ }^{17}$ that the minimal units required for full affinity binding are RPTP $\sigma$ Ig1-3 and TrkC LRRIg1 (Supplementary Fig. 5a,b; Supplementary Table 1). $\mathrm{RPTP} \sigma$ Ig1-2 does retain TrkC binding, although with an approximately fivefold-reduced affinity compared with RPTP $\sigma$ Ig1-3 $\left(K_{\mathrm{d}}=2.4 \mu \mathrm{M}\right.$ versus $551 \mathrm{nM}$, Supplementary Fig. $\left.5 \mathrm{a}\right)$. TrkC LRRIg1 had also previously been shown to be the minimal unit required for the synaptogenic activity of $\mathrm{TrkC}^{17}$. To facilitate crystallization, a chicken TrkC construct (TrkC LRRIg1 $1_{\text {cryst }}$ ) was generated, which removed putative sites of $\mathrm{N}$-linked glycosylation and residues 63-77, a predicted disordered loop (Supplementary Fig. 6). A $2.5 \AA$ crystal structure of a chicken RPTP $\sigma$ Ig1-2:TrkC LRRIg1 $1_{\text {cryst }}$ complex was determined, revealing a 1:1 stoichiometry (Fig. 2b; Table 1; Supplementary Fig. 5c), in agreement with results from multi-angle light scattering (MALS) analysis (Supplementary Fig. 5d). The structure is consistent with a trans RPTP $\sigma: T r k C$ complex spanning the synaptic cleft (Fig. 2b). The V-shaped RPTP $\sigma$ Ig1-2 module contacts an extended TrkC surface consisting of the LRR convex face and Ig1 domain, with a buried surface area of $1,093 \AA^{2}$ per molecule (Fig. 2b).

Three major contact sites constitute the protein-protein interface in the complex crystal structure: site 1, RPTP $\sigma$ Ig1:TrkC Ig1 (Fig. 2c; Supplementary Fig. 7); site 2, RPTP $\sigma$ Ig1:TrkC LRRIg1 inter-domain region (Fig. 2d; Supplementary Fig. 7) and site 3, RPTP $\sigma$ Ig2:TrkC LRR (Fig. 2e; Supplementary Fig. 7). Electrostatic interactions involving RPTP $\sigma$ residues R96 and R99 and TrkC residues D240 and D242 dominate interactions at site 1 (Fig. 2c). Intriguingly, R96 and R99 form part of the extended positively charged surface on RPTP $\sigma$ Ig1 (ref. 14) and are absolutely required for RPTP $\sigma$ interactions with HSPGs ${ }^{10,14}$, suggesting that TrkC and proteoglycans may compete for binding to RPTP $\sigma$. At site 2, Q75 in RPTP $\sigma$, interacts with TrkC residues E287 and Q148, while the side chains of RPTP $\sigma$ E78 and TrkC R121 form a salt bridge (Fig. 2d). Site 3 centres on
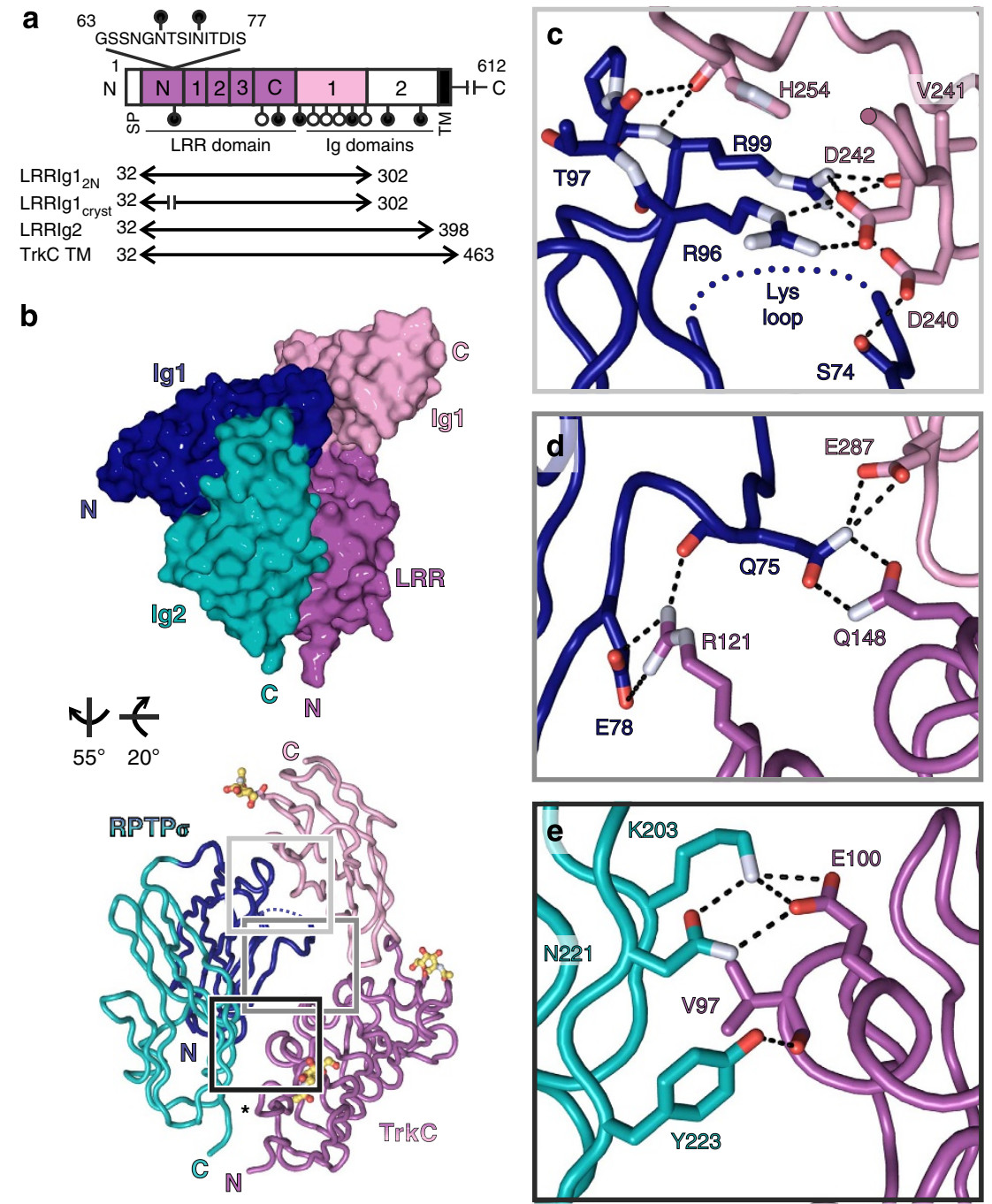

Figure 2 | Trans-synaptic RPTPo:TrkC complex crystal structure. (a) TrkCTK- (non-catalytic isoform) domain organization. LRR, leucine-rich repeat region ( $\mathrm{N}, \mathrm{N}$-terminal cysteine-rich region; 1-3, leucine-rich repeats; $\mathrm{C}, \mathrm{C}$-terminal cysteine-rich region). Putative $\mathrm{N}$-linked glycosylation sites, lollipops; filled lollipops remain in LRRIg1 cryst construct. (b) Space-filled and tube representations of chicken RPTP $\sigma$ Ig1-2:TrkC LRRIg1 cryst crystal structure. $\mathrm{N}$-linked glycans in atom representation. Disordered RPTP $\sigma$ Lys-loop, blue dotted line; TrkC LRRIg1 $1_{\text {cryst }}$ amino-acid residue 62-78 junction, asterisk. (c-e) Detailed view of bonding interactions at RPTP $\sigma: T r k C$ interface for binding sites 1-3. Corresponding electron density is illustrated in Supplementary Fig. 7. Potential electrostatic and hydrogen bonds, black dashed lines; oxygen atoms, red; nitrogen atoms, bluewhite. 
a K203-E100 salt bridge between RPTP $\sigma$ and TrkC residues, respectively (Fig. 2e).

These three interaction sites are consistent with RPTP $\sigma$ binding TrkC, but not TrkA or $\operatorname{TrkB}^{17}$; of the six predominantly charged (D240, D242, E287, Q148, R121 and E100) TrkC residues observed to have side-chain-mediated RPTP $\sigma$ interactions, only E287 is conserved across the other Trk family members (Supplementary Fig. 6). Although the key $\mathrm{RPTP} \sigma$ residues discussed above are conserved in human RPTP $\delta$ and LAR (Supplementary Fig. 1), the specificity of TrkC binding for RPTP $\sigma$ can be rationalized through closer inspection of type IIa RPTP sequence alignments and the chicken RPTP $\sigma$ Ig12:TrkC LRRIg $1_{\text {cryst }}$ crystal structure (Fig. 3a). At site 1 , we hypothesized that substitution of P98 (in RPTP $\sigma$ ) to H98 in RPTP LAR, would result in a movement of the carbonyl group of T97 to ease the cis-conformation of residue 98 to trans (see PDB ID: 2YD5), disrupting interaction with the TrkC H254 carbonyl group. At site 2, we anticipated that substitution of S74 (in $\mathrm{RPTP} \sigma$ ) to N74 in RPTP $\delta$ would result in the loss of this residue's interaction with TrkC D240. To validate these predictions, RPTP $\sigma$ Ig1-3 P97V + T98H (LAR-like Ig1-3) and $\mathrm{N} 73 \mathrm{~S}+\mathrm{S} 74 \mathrm{~N}(\mathrm{RPTP} \delta$-like Ig1-3) proteins were generated, which indeed displayed LAR- and RPTP $\delta$-like binding to TrkC in SPR analyses (Fig. 3b,c; Supplementary Fig. 8a,b; Supplementary Table 1).

Validation of the RPTP $\sigma$ :TrkC binding mode. To confirm the contribution of the RPTP $\sigma:$ TrkC interaction sites, we introduced point mutations into the interfaces on either protein (Fig. 4a) and measured the resulting dissociation constants $\left(K_{\mathrm{d}}\right)$ using SPR. As predicted, mutations in either TrkC (D240A + D242A) or RPTPF (R96A + R99A, affecting the glycosaminoglycan (GAG)binding arginine residues), completely abolished both human and chicken RPTP $\sigma$ binding to mouse and chicken TrkC, respectively
(Fig. 4b,c; Supplementary Fig. 8c-f; Supplementary Table 1). The RPTP $\sigma$ Y223S mutation was designed to disrupt binding at site 3 by introducing an $N$-linked glycosylation site at N221, and RPTP $\sigma: T r k C$ binding was indeed ablated (Fig. 4c; Supplementary Fig. 8e,f; Supplementary Table 1). In agreement with the structural and biophysical data, TrkC transmembrane (TrkC TM) D240A + D242A expressing COS-7 cells, unlike wild-type TrkC TM expressing positive controls, were unable to induce presynaptic differentiation in co-cultured rat hippocampal neurons despite comparable levels of cell surface expression (Fig. 4d; Supplementary Fig. 9).

We had noted that the interaction affinity between engineered proteins used for RPTP $\sigma: \operatorname{TrkC}$ complex crystallization (chicken RPTP $\sigma$ Ig1-3 and TrkC LRRIg $1_{\text {cryst }}$ ) was some 20 -fold lower following the TrkC 63-77 loop deletion ( $K_{\mathrm{d}} 4.8 \mu \mathrm{M}$ versus 216 nM; Fig. 5a; Supplementary Fig. 8g; Supplementary Table 1). We therefore explored the possible contribution of the 63-77 loop to the RPTP $\sigma: T r k C$ interaction by engineering a construct termed TrkC LRRIg $1_{2 Q}$, comprising the full sequence but still removing two predicted $N$-linked glycosylation sites by introducing N68Q and N72Q mutations (Supplementary Fig. 6). This construct did indeed bind RPTP $\sigma$ with enhanced affinity (Fig. 5a; Supplementary Fig. 8g,h; Supplementary Table 1), while TrkC $\mathrm{TM}_{2 \mathrm{Q}}$ induced comparable levels of presynaptic differentiation in co-cultured rat hippocampal neurons to wild-type TrkC TM (Fig. 4d). We determined the $3.05 \AA$ crystal structure of this chicken RPTP $\sigma$ Ig1-3:TrkC LRRIg1 $1_{2 Q}$ complex in an attempt to visualize this additional, fourth, binding site (Table 1). Crystals grew in a new space group, P1, with three RPTP $\sigma: T r k C$ complexes in the asymmetric unit (a.s.u.). These align very closely with the two complexes/a.s.u. observed in the previous P2 structure (Fig. 5b; root mean squared deviation between 446 equivalent $\mathrm{C} \alpha$ residues of the $\mathrm{P} 2$ complex1 relative to $\mathrm{P} 2$ complex2 and P1 complexes1-3 was calculated to be $0.82 \AA$, $0.83 \AA, 0.80 \AA$ and $0.70 \AA$, respectively), providing additional a
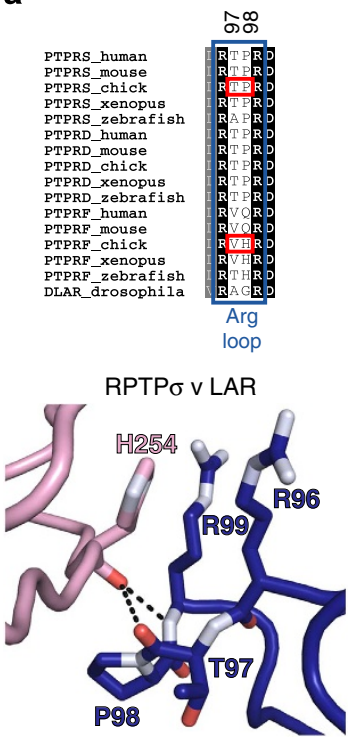

$5 \pi$
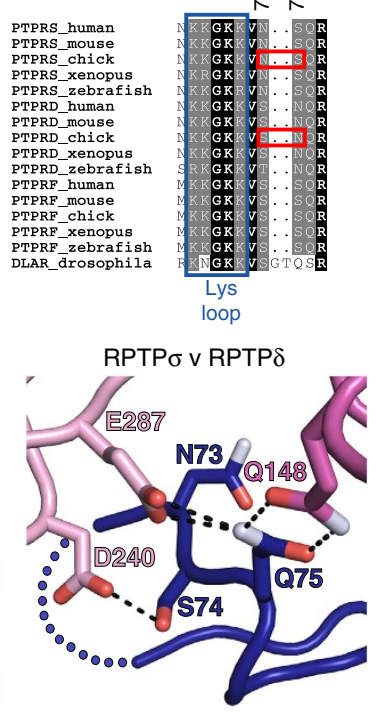

b
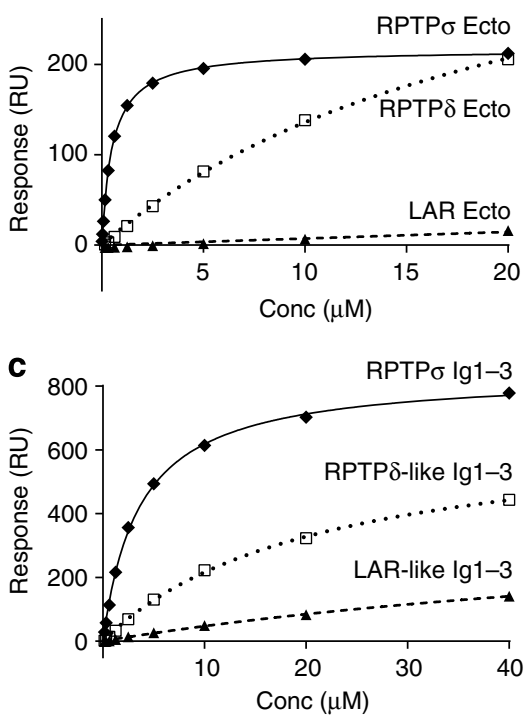

Figure 3 | TrkC binding preferences for type Ila RPTP family members. (a) Type Ila RPTP sequence alignments and detailed views of the RPTP $\sigma:$ TrkC crystal structure at binding site 1 (left) and binding site 2 (right). Blue boxes indicate RPTP $\sigma$ residues required for proteoglycan binding, and red boxes highlight key sequence differences conferring TrkC-binding specificity. Colour scheme as in Fig. 2: dark blue, RPTP $\sigma$ Ig1; pink, TrkC LRR; magenta, TrkC Ig1. (b) SPR analysis of human type Ila RPTP ectodomains binding to immobilized mouse TrkC LRRIg1. Measured binding values: RPTP $\sigma$ Ecto, $K_{\mathrm{d}}=516 \mathrm{nM}$ and $B_{\max }=217 \mathrm{RU} ; \mathrm{RPTP} \delta$ Ecto, $K_{\mathrm{d}}>22 \mu \mathrm{M}$ and $B_{\max }>433 \mathrm{RU}$; RPTP LAR, $K_{\mathrm{d}}$ and $B_{\max }$ not determined. (c) SPR analysis of chicken TrkC LRRIg1 binding to immobilized chicken RPTP $\sigma$ lg1-3, RPTP $\sigma$ N73S + S74N (RPTP - -like) lg1-3 and RPTP $\sigma$ P97V + T98H (LAR-like) lg1-3. Measured binding values: RPTP $\sigma$ lg1-3, $K_{d}=3.5 \mu \mathrm{M}$ and $B_{\max }=837 \mathrm{RU}$; RPTPS-like Ig1-3, $K_{\mathrm{d}}>21 \mu \mathrm{M}$ and $B_{\max }>674 \mathrm{RU}$; RPTP LAR-like Ig1-3, $K_{\mathrm{d}}$ and $B_{\max }$ not determined. For sensograms see Supplementary Fig. $8 a, b$. 


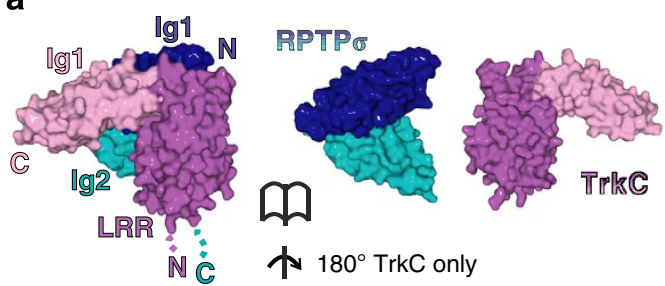

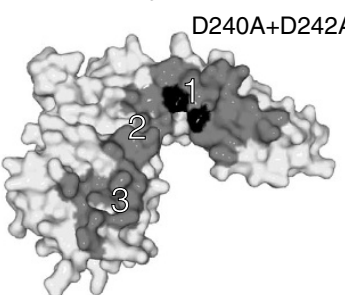
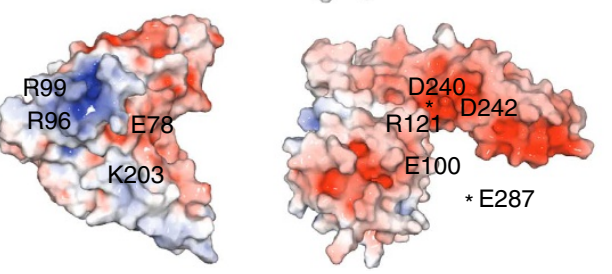

b

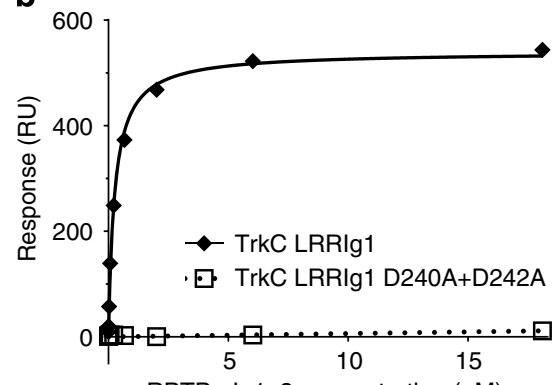

RPTP $\sigma$ Ig1-3 concentration $(\mu \mathrm{M})$

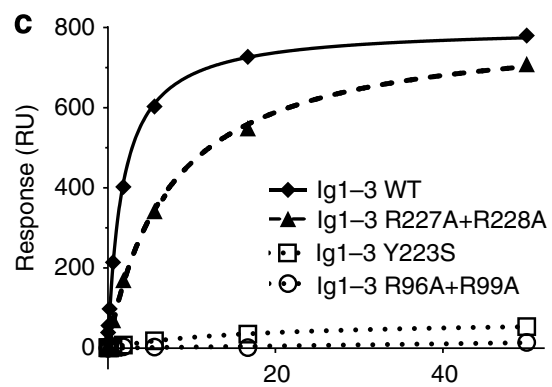

TrkC LRRIg1 concentration $(\mu \mathrm{M})$

d

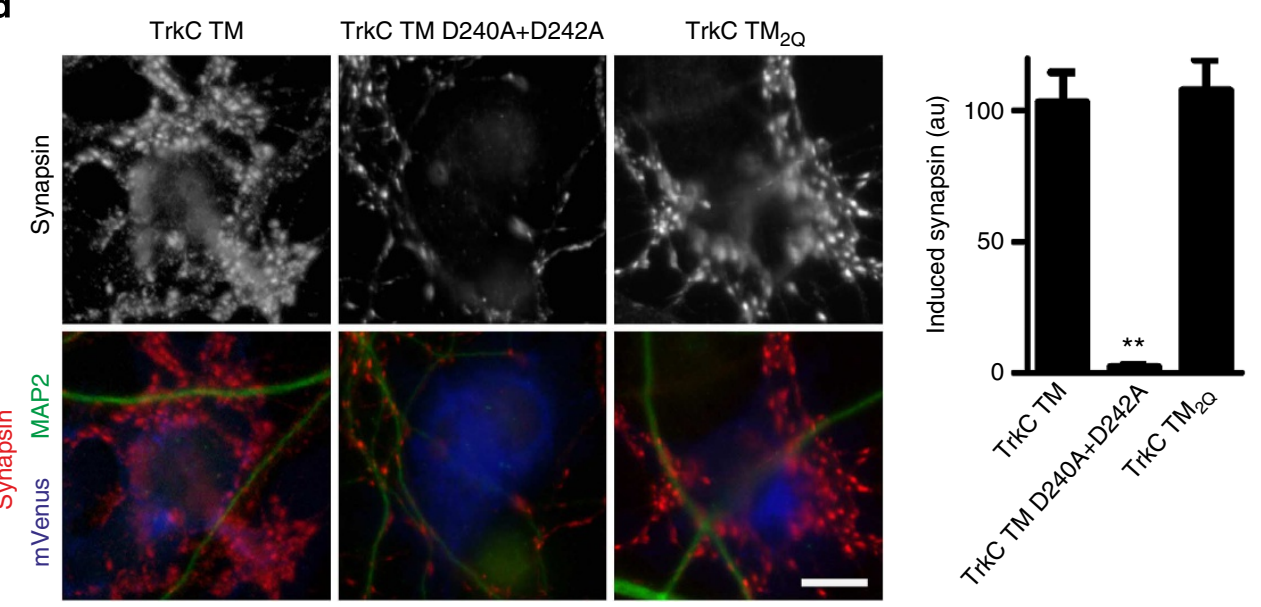

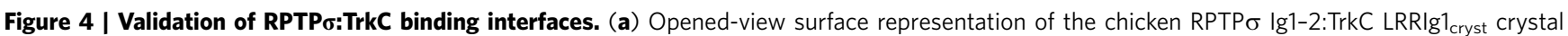
structure. RPTP $\sigma$ and TrkC interface residues are coloured grey and interface mutants used in biophysical and cellular assays are highlighted in black (middle panel). Binding sites 1-4 are labelled. RPTP $\sigma$ and TrkC are coloured by electrostatic potential (bottom panel) from red ( $-8 \mathrm{kT} / \mathrm{e}$ ) to blue $(+8 \mathrm{kT} / \mathrm{e})$, illustrating complementary charged patches at binding sites 1-3 (note that the basic RPTP $\sigma$ 'Lys-loop' residues 68-71 are absent in the RPTPo:TrkC complex crystallographic model). (b) SPR analysis of human RPTP $\sigma$ lg1-3 binding to immobilized mouse TrkC LRRIg1 and TrkC LRRIg1 $\mathrm{D} 240 \mathrm{~A}+\mathrm{D} 242 \mathrm{~A}$. Measured binding values: TrkC LRRIg1, $K_{\mathrm{d}}=258 \mathrm{nM}$ and $B_{\max }=540 \mathrm{RU}$; TrkC LRRIg1 D240A + D242A, $K_{\mathrm{d}}$ and $B_{\max }$ not determined. (c) Mouse TrkC LRRIg1 binding to immobilized human RPTP $\sigma$ lg1-3 WT, R96A + R99A, Y223S and R227A + R228A. Measured binding values: RPTP $\sigma$ Ig1$3 \mathrm{WT}, K_{\mathrm{d}}=1.8 \mu \mathrm{M}$ and $B_{\max }=802 \mathrm{RU} ; \mathrm{lg} 1-3 \mathrm{R} 227 \mathrm{~A}+\mathrm{R} 228 \mathrm{~A}, K_{\mathrm{d}}=7 \mu \mathrm{M}$ and $B_{\max }=806 \mathrm{RU} ; \mathrm{Ig} 1-3 \mathrm{R} 96 \mathrm{~A}+\mathrm{R} 99 \mathrm{~A}$ and $\mathrm{Y} 223 \mathrm{~S}, K_{\mathrm{d}}$ and $B_{\max }$ not determined. For sensograms see Supplementary Fig. 8c-f. (d) Induced synapsin clustering in rat hippocampal neurons by TrkC TM (WT), TrkC TM_D240A + D242A and TrkC TM2Q expressing COS-7 cells. Analysis of variance, $P<0.0001 ;{ }^{\star} * P<0.001$ compared with TrkC TM by post hoc Bonferroni's multiple comparison test, $n=26$ cells from two experiments. Scale bar, $10 \mu$ M. Relative cell surface expression levels are shown in Supplementary Fig. 9.

support for the relevance of the observed RPTP $\sigma:$ TrkC interaction mode. The site 4 interface is not well resolved in this $3.05-\AA$ resolution structure, although we do observe an additional TrkC helix (formed by residues 69-80), which interacts with RPTP $\sigma$ Ig2 predominantly via potential packing of the I73 side chain against a hydrophobic region consisting of RPTP $\sigma$ residues V144, Y223 and TrkC L56, T74 and L101 (Fig. 5c,d). The RPTPo Ig2-Ig3 linker (V226-A230) and the Ig3 domain also lack wellordered electron density in this structure. However, a R227A+ R228A double mutation reduced RPTP $\sigma: T r k C$ binding (Fig. 4c; Supplementary Fig. 8e,f; Supplementary Table 1), as did insertion of the meB mini-exon between R225 and V226 in SPR-binding assays, supporting the involvement of the RPTP $\sigma$ Ig2-Ig3 linker in the auxilary binding site 4 (Supplementary Fig. 8i; Supplementary Table 1).

HSPGs compete with TrkC for RPTPo binding. The overlap of the TrkC- and GAG-binding sites on RPTP $\sigma$, prompted us to investigate the notion that proteoglycan competition with TrkC has the potential to modulate RPTP $\sigma$ function in synaptogenesis. Initially, we tested whether soluble HS or the HS-mimetic 


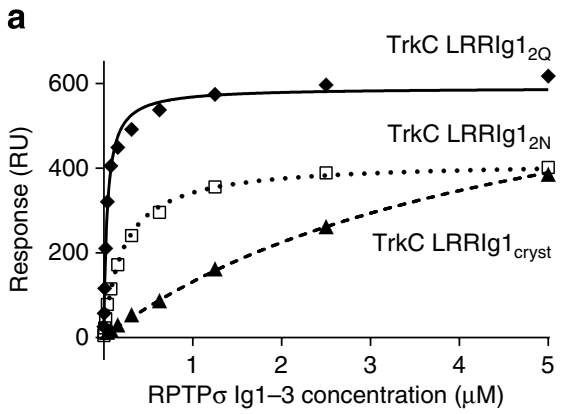

C
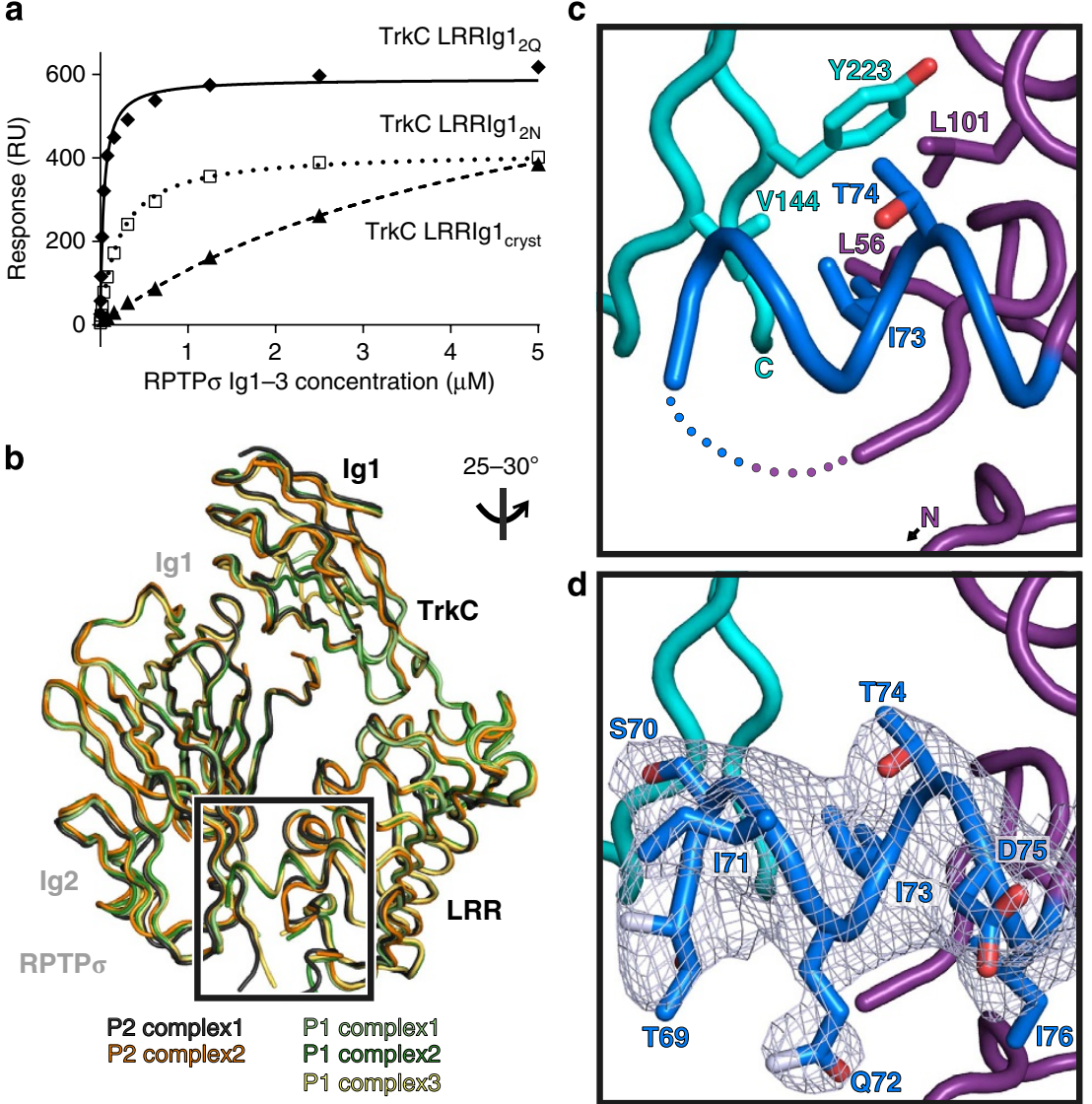

Figure 5 | Characterization of the potential accessory RPTPa:TrkC-binding site 4. (a) SPR analysis of chicken RPTP $\sigma$ lg1-3 binding to immobilized chicken TrkC LRRIg1 $1_{\text {cryst }}, \mathrm{LRRI}_{2 \mathrm{~N}}$ and LRRIg1 $1_{2 \mathrm{Q}}$. Measured binding values: TrkC LRRlg1 $1_{\text {cryst }}, K_{\mathrm{d}}=4.8 \mu \mathrm{M}$ and $B_{\max }=761 \mathrm{RU} ; \mathrm{LRRIg} 1_{2 \mathrm{~N}}, K_{\mathrm{d}}=216 \mathrm{nM}$ and $B_{\max }=416 \mathrm{RU} ; \mathrm{LRRIg} 1_{2 \mathrm{Q}}, K_{\mathrm{d}}=38 \mathrm{nM}$ and $B_{\max }=590 \mathrm{RU}$. For sensograms see Supplementary Fig. 8g-h. (b) Alignment of the two RPTP $\sigma:$ TrkC complexes observed in the chicken RPTP $\mathrm{Ig} 1-2:$ TrkC LRRIg1 $1_{\text {cryst }}$ (P2 space group) and three in the chicken RPTP $\sigma$ lg1-3:TrkC LRRIg1 $2 \mathrm{Q}$ (P1 space group) crystal structures. The orientation of the structures is identical to Fig. $2 \mathrm{~b}$ (lower panel). (c) Additional features observed in complex 1 from the RPTP $\sigma$ lg1-3:TrkC LRRIg1 $_{2 Q}$ crystal structure. Residues within the 63-77 loop that were not present in the P2 crystal structure are coloured blue and the remaining missing residues are indicated by dotted lines. View rotated relative to $\mathbf{b}$ as indicated. TrkC LRR, magenta; RPTP $\sigma$, cyan. (d) SigmaA weighted $2 F_{0}-F_{c}$ electron density map (grey) contoured at $1 \sigma$ and carved at $2.2 \AA$ around loop residues $69-76$.

heparin-dp10 could inhibit the interactions between TrkC and either wild-type $\mathrm{RPTP} \sigma$ or a quadruple $\mathrm{K} 67 \mathrm{~A}+\mathrm{K} 68 \mathrm{~A}+$ $\mathrm{K} 70 \mathrm{~A}+\mathrm{K} 71 \mathrm{~A}$ mutant $(\mathrm{RPTP} \sigma \mathrm{Ig} 1-3 \Delta \mathrm{K})$, which precludes $\mathrm{GAG}$ binding $^{14}$ while retaining wild-type TrkC binding (Fig. 6; Supplementary Fig. 10a,b; Supplementary Table 1). Increasing concentrations of HS or heparin-dp10 were able to inhibit the binding of RPTP $\sigma$ Ig1-3 WT, but not RPTP $\sigma \operatorname{Ig} 1-3 \Delta \mathrm{K}$ to immobilized TrkC LRRIg1 (Fig. 6a; Supplementary Fig. 10a,b; Supplementary Table 1). To investigate the TrkC versus GAG competition in a cellular setting, we added heparin-dp10 to cocultures of TrkC TM expressing COS-7 cells and rat hippocampal neurons. Induced presynaptic differentiation in the neurons decreased by twofold compared with mock-treated co-cultures upon heparin-dp10 addition (Fig. 6b,c). Furthermore, treatment of co-cultures with a mixture of heparinases I, II and III, to digest heparan sulphate GAGs, significantly enhanced presynaptic induction by $\operatorname{TrkC} \mathrm{TM}$ (Fig. 6b,c), suggesting that native HSPGs may limit synapse development through RPTP $\sigma: T r k C$, by direct competition for binding (Fig. 6d). In contrast to TrkC, the interaction of RPTP $\sigma$ with another trans-synaptic protein ligand, NGL-3, reported to bind at the FN1-2 domains, appears insensitive to proteoglycans. Neither heparin-dp10 nor heparinase treatment affected NGL-3-induced synaptogenesis in the co-culture system (Fig. 6b,c), and RPTP $\sigma: N G L-3$ binding in SPR assays showed no major reduction upon HS addition (Supplementary Fig. 10c,d; Supplementary Table 1).

\section{Discussion}

While all three vertebrate type IIa RPTP family members bind NGL-3 (refs 18,27) and interleukin-1 receptor accessory protein $^{19}$, and both RPTP $\sigma$ and RPTP $\delta$ bind to Slitrk1 and Slitrk2 (refs 20,28), the IL-1 receptor accessory protein-like 1 (IL1RAPL1) interacts predominantly with RPTP $\delta^{29,30}$ and the receptor protein tyrosine kinase TrkC interacts with RPTP $\sigma^{17}$. We therefore used this latter protein-protein interaction as our exemplar for trans-synaptic RPTP $\sigma$ action. RPTP $\sigma$ and TrkC exhibit broad and overlapping expression patterns in the adult nervous system ${ }^{18,21,31-33}$. Multiple ligand interactions and signalling pathways are disrupted in RPTP $\sigma$ - and TrkCdeficient mice though, making assessment of any overlap in phenotypes difficult ${ }^{34-37}$. A direct comparison of the effect of either TrkC knockdown ${ }^{17}$ or RPTP $\sigma$ knockout ${ }^{38,39}$ upon synapse structure and number in vivo, is similarly complicated by the parallel role of RPTP $\sigma$ in regulating axon sprouting. The detailed explanation of RPTP $\sigma: T r k C$ specificity that we offer in this study provides the information to enable the design of new experiments to dissect the precise contribution of this interaction to 


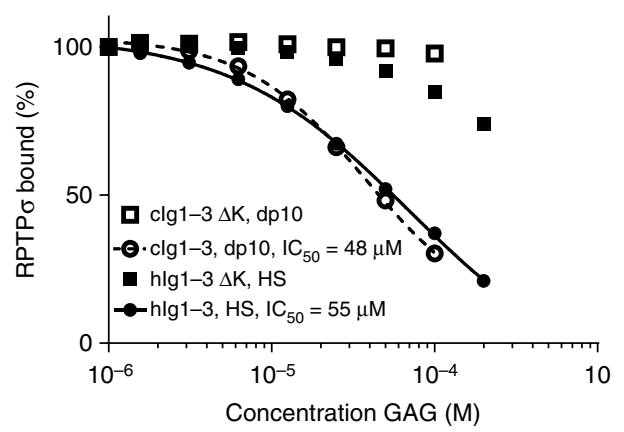

b
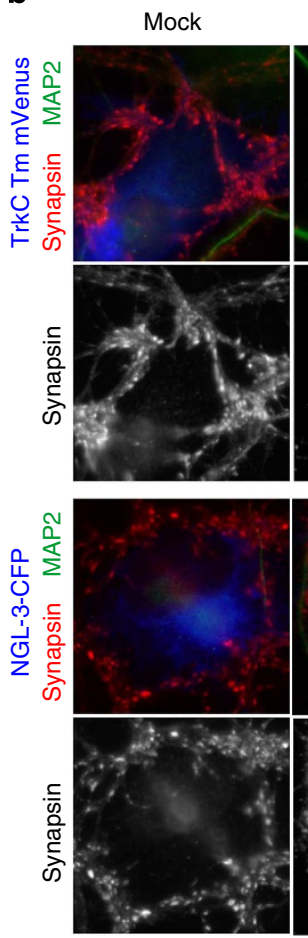

$\mathrm{dp} 10$
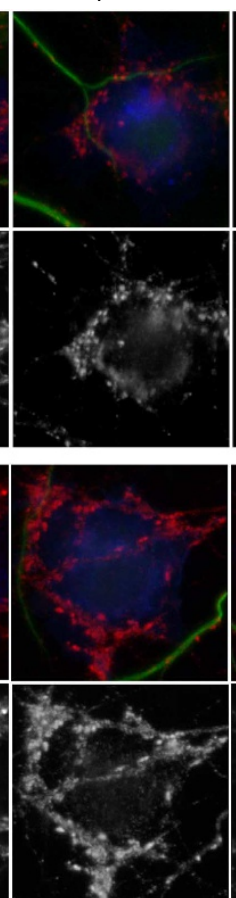

Heparinases
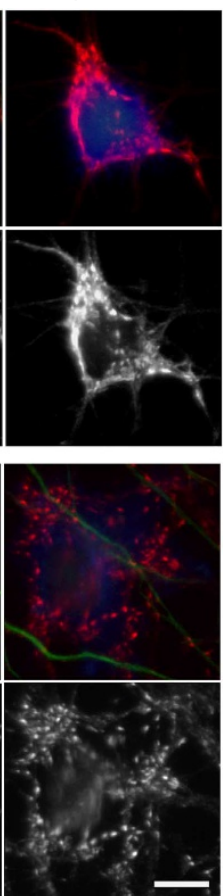

C

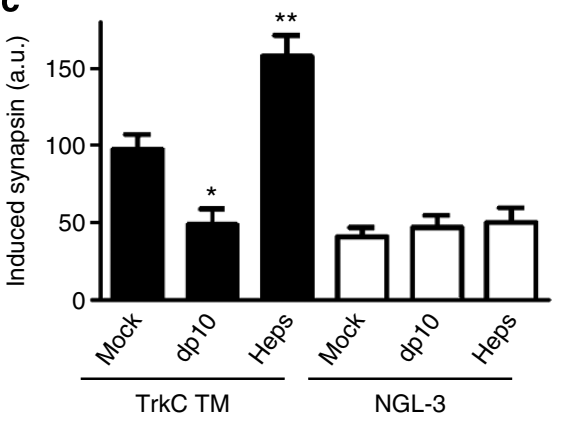

d
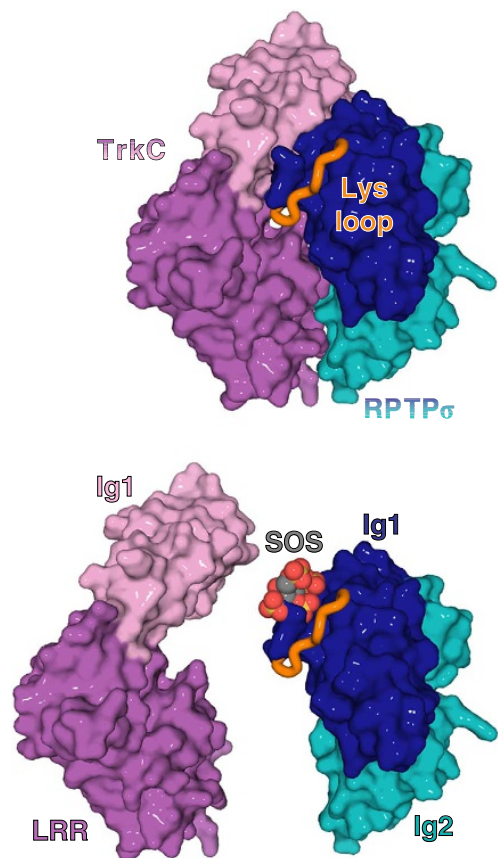

Figure 6 | GAG-mediated inhibition of synaptic RPTPo:TrkC interaction and function. (a) SPR analysis of human RPTP $\sigma$ lg1-3 and RPTP $\sigma$ lg1-3 $\triangle K$ binding to immobilized mouse TrkC LRRIg1 in the presence of increasing concentrations of HS or heparin-dp10. For sensograms see Supplementary Fig. 10a,b. (b,c) Induced synapsin clustering in rat hippocampal neurons by COS-7 cells expressing TrkC TM or NGL-3 in the presence of heparin-dp10, heparinases (Heps) or mock control. Analysis of variance $P<0.0001,{ }^{\star} P<0.01$ and ${ }^{\star \star} P<0.001$ compared with TrkC TM mock, whereas NGL-3 heparin-dp10 or heparainase groups were not significantly different from NGL-3 mock by post hoc Bonferroni's multiple comparison test, $n=16-26$ cells from two experiments. Scale bar, $10 \mu \mathrm{M}$. (d) Illustration of the partial overlap between GAG- and TrkC-binding sites on RPTP $\sigma$. Top panel: the RPTP $\sigma$ :TrkC complex, rotated $180^{\circ}$ around the y axis relative to Fig. 2b. Lower panel: sucrose octasulphate (SOS, grey/red) is modelled in the RPTP $\sigma$ GAG-binding site, an equivalent location to that observed in the LAR:SOS co-crystal structure (which is homologous with RPTPG, PDB ID: 2YD8). SOS (or GAG) binding can out-compete the TrkC interaction with RPTPo.

synaptogenesis in vivo. The locations of RPTP $\sigma$ and TrkC are primarily reported as pre- and postsynaptic, respectively ${ }^{8,9}$, and we depict them as such in our model (Fig. 7). However, there is evidence that this may be too simplistic, for example, the type IIa RPTPs have also been reported in postsynaptic compartments ${ }^{7}$. The extent of flexibility we observe for the RPTP $\sigma$ ectodomain, suggests that the RPTP $\sigma: T r k C$ binding mode revealed by our crystal structures may also mediate cis interactions in the event of co-localization of the two receptors at the same cell surface.

Our data reveal several key properties that fit RPTP $\sigma$ for its dual role as a synaptic signalling hub and a promoter of neuronal growth. During axonal extension, RPTP $\sigma$ interacts with proteoglycan molecules through its $\mathrm{N}$-terminal Ig1 domain, the clustering properties of HSPGs promoting growth cone motility $^{14}$. The RPTPo ectodomain architecture described here indicates that a range of conformations can be explored, permissive of cis interactions at the axonal surface and trans interactions to the general extracellular milieu including the basement membrane (Fig. 7a). Indeed, the length of the RPTP $\sigma$ ectodomain may be important to extend the HSPG-binding site beyond a saturating layer of cis interactions at the same cell surface, similar to the sialic acid-binding Siglec family of cell surface receptors where a lengthy ectodomain is required to escape the inhibitory glycocalyx ${ }^{40}$. At the transition from extension to synaptogenesis, the postsynaptic neuronal surface presents an array of additional RPTP $\sigma$ ligands (Fig. $7 \mathrm{~b})^{8,9}$. Synapse formation and development requires the selection of an appropriate subset of binding partners. This involves a simple kinetic competition for binding, governed by inter-molecular affinity and interaction site accessibility. Our analyses suggest that 
this selection may be choreographed by the interplay of binding site location and conformational flexibility in the RPTP $\sigma$ ectodomain. Structural comparison of the RPTP $\sigma: \operatorname{TrkC}$ complex with our previously reported interaction mode for type IIa RPTP:GAGs ${ }^{14}$ shows partially overlapping binding sites (Fig. 6d). Thus, during synapse formation, and the shift from axonal growth to synaptic stability, postsynaptic TrkC must outcompete proteoglycans for RPTP $\sigma$ binding, simultaneously providing an adhesive trans interaction and extinguishing the RPTP $\sigma$-clustering activity of HSPGs (Fig. 7b). Neuronal and gliareleased proteoglycans continue to play important roles at synapses ${ }^{41,42}$, however, their contribution may involve other trans-synaptic interactions, for instance other type IIa RPTP family members or LRRTM4 (refs 43,44).

Alternative splicing has been shown previously to control the specificity of trans-synaptic interactions of the type IIa $\mathrm{RPTPs}^{8,17,20,28,29}$. The impact of alternative splicing at RPTP $\sigma$ mini-exon site meB on TrkC binding affinity provides a potential rheostat by which to fine-tune the balance between competing RPTP $\sigma$ ligands, an analogous feature to neurexin splice site 4 control of ligand interactions at the synaptic cleft ${ }^{8,45,46}$. The meA mini-exon is absent from all the RPTP $\sigma$ constructs used in this study, but its insertion into the Ig2 $\beta D-\beta E$ loop ( $\beta 11-\beta 12$ loop in Supplementary Fig. 1) is unlikely to affect binding of RPTP $\sigma$ to TrkC, as it would lie on the opposite face to the TrkC-binding interface. Both meA and meB sites are remote from the GAGbinding surface on RPTP $\sigma$ Ig1 (ref. 14) and therefore neither insertion is expected to modulate binding of RPTP $\sigma$ to proteoglycans.

Trans-synaptic binding to TrkC will limit the conformational freedom of the RPTP $\sigma$ ectodomain. This reduces the entropic

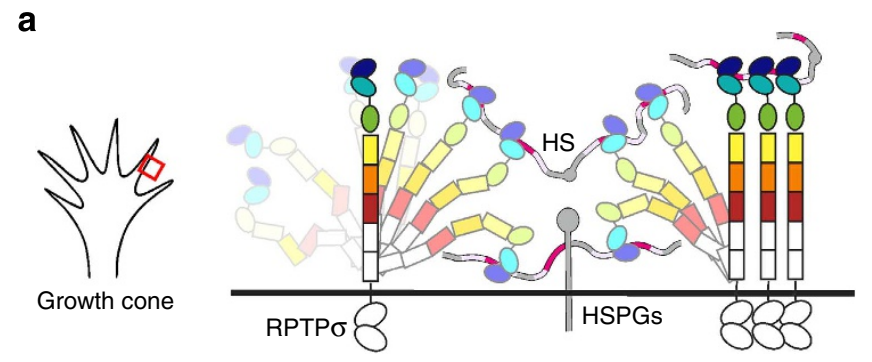

b
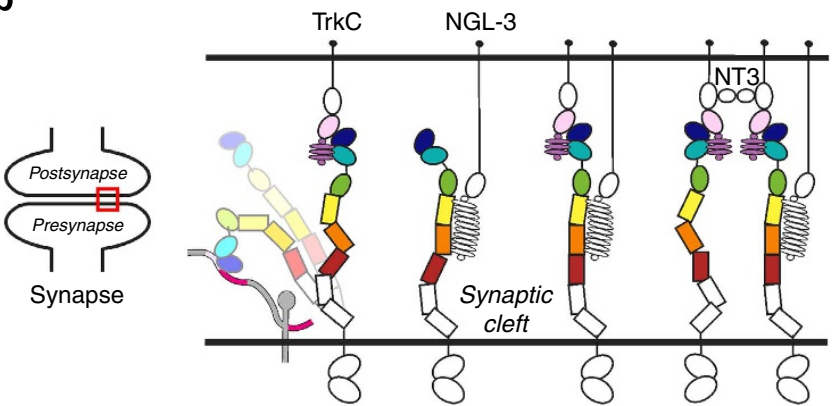

Figure 7 | Model illustrating flexible RPTP $\sigma$ ectodomain sampling of extracellular ligands. (a) At the growth cone, RPTP $\sigma$ interacts with cell surface and basal membrane proteoglycans to mediate axonal extension. (b) Upon contact with target cells, to shift to the role of synaptic organizer, RPTP $\sigma$ adopts elongated conformations to protrude from the presynaptic proteoglycan haze and bind postsynaptic ligands such as TrkC and NGL-3. Subsequent independent or coordinated interactions with additional synaptic ligands are shown. Red boxes (left hand panels) indicate growth cone (a) or synapse (b) regions that are illustrated in the right-hand cartoons. penalty for binding at other sites on RPTP $\sigma$, an effect which can potentiate the formation of cell surface assemblies involving multiple receptor interactions ${ }^{47}$. For RPTP $\sigma$ function at the synapse this may facilitate cooperative binding of TrkC and NGL3 , as these two binding sites are separate ${ }^{18}$ (Fig. 7b). TrkC can also bind the NT-3 neurotrophin, a modulator of synaptic transmission, at the second Ig domain ${ }^{48}$ (Fig. $7 \mathrm{~b}$ ), which adds a further, distinctive stoichiometry to this network of transsynaptic interactions, by specifically triggering dimerization of TrkC and hence RPTP $\sigma$. Other soluble modulators, such as the astrocyte-derived HSPGs glypican-4 and glypican-6, may provide an alternative strategy to regulate this system ${ }^{41}$. The stoichiometry and architecture of higher-order trans-synaptic complexes, and their impact on RPTP $\sigma$ enzymatic activity, remain important questions to address in the future. Formally, we cannot yet exclude a scenario where the simple kinetics of competition between ligands may be sufficient to explain the phenotypes observed in our cellular assays. However, given the geometrical constraints within which the RPTP $\sigma$ ectodomain has to operate at points of cellular contact, with ligands present in both cis and trans orientations, it is very likely that the RPTP $\sigma$ ectodomain flexibility is required in a physiological context.

A series of proteolytic processing events have been reported for the type IIa RPTPs at the cell surface, involving initial shedding of the ectodomain, prior to release of the intracellular catalytic domain $s^{49}$. In our crystallization trials, we have potentially identified a further receptor-cleavage site at the consensus furinlike protease motif RVRR on the Ig2-Ig3 linker, which would be disrupted in RPTP $\sigma$ isoforms containing mini-exon meB. Cleavage of either RPTP $\sigma$ Ig1-2 or ectodomain fragments would first decouple the receptor phosphatase activity from regulation via both proteoglycan and TrkC binding, and second the released soluble RPTP $\sigma$ fragments would be able to compete with the remaining intact receptors for binding to these same ligands. Knockout of the Drosophila type IIa RPTP dLAR, can be rescued by reintroduction of a catalytically inactive receptor, but not by a dLAR construct lacking the second inactive phosphatase domain $(\mathrm{D} 2)^{50}$. It remains to be determined whether RPTP $\sigma$ ectodomain-ligand interactions may regulate the availability of RPTP $\sigma$ D2 for binding downstream intracellular partners ${ }^{8,9}$, but any such mode of regulation would also be ablated by extracellular RPTP $\sigma$ cleavage events.

In conclusion, our results suggest how the RPTP $\sigma$ nexus utilizes a series of filtering mechanisms to discriminate between binding options, and ultimately integrate the signalling inputs essential for the transition from neuronal growth to synapse organization. Rigidity has been demonstrated to be central for the comparatively simpler adhesion molecule function ${ }^{24-26}$. In contrast, the properties of RPTP $\sigma$ described here, prompt the notion of how ectodomain flexibility can allow a cell surface receptor to integrate a broad spectrum of ligand interactions into distinct functional outcomes.

\section{Methods}

Construct design and cloning. Human RPTP $\sigma$ Ig1-3, Ig1-FN3 and sEcto pHLsec constructs were reported previously ${ }^{14}$. The chick RPTP $\sigma$ Ig1-3 construct (amino acids 29-316, NCBI Ref. Seq. NM_205407.1) was cloned into pHLsec and pHLAvitag3 vectors ${ }^{51}$. A series of chick RPTP $\sigma$ Ig1-3 mutant constructs were generated by PCR: K67A + K68A + K70A + K71A (chick RPTP $\sigma$ Ig1-3 $\Delta \mathrm{K}$ ), $\mathrm{N} 73 \mathrm{~S}+\mathrm{S} 74 \mathrm{~N}, \mathrm{R} 96 \mathrm{~A}+\mathrm{R} 99 \mathrm{~A}, \mathrm{P} 97 \mathrm{~V}+\mathrm{T} 98 \mathrm{H}, \mathrm{K} 203 \mathrm{~A}, \mathrm{Y} 223 \mathrm{~S}$ and R227A + R228A and subsequently cloned into the pHL-Avitag3 vector. Human RPTP $\sigma$ Ig1-3 $\mathrm{K} 68 \mathrm{~A}+\mathrm{K} 69 \mathrm{~A}+\mathrm{K} 71 \mathrm{~A}+\mathrm{K} 72 \mathrm{~A}$ (human RPTP $\sigma$ Ig1-3 $\Delta \mathrm{K}$ ), R97A + R100A, Y224S and R228A + R229A C-terminal Avitag constructs were similarly constructed. Human RPTP $\delta$ sEcto (amino acids 21-833, NCBI Ref. Seq. BC106713.1), and human RPTP LAR Ecto (amino acids 30-1,260, NCBI Ref. Seq. NM_002840.3) were also cloned into the pHL-Avitag3 vector.

A synthetic clone for chick TrkC was commercially synthesized (Source Bioscience); to include amino-acid residues 32-302 (NCBI Ref. Seq. 
NM_205169.1), but lack residues 63-77 and include the following point mutations: N163Q, N232Q, N259Q, N267Q and N294Q, to reduce the number of $N$-linked glycosylation sites. This construct (chick TrkC LRRIg1 $1_{\text {cryst }}$ ) was cloned into the pHL-Avitag3 vector. A series of further chick TrkC LRRIg1 mutant constructs were generated by PCR: D240A + D242A, re-addition of residues 63-77 (chick TrkC LRRIg $1_{2 \mathrm{~N}}$ ) and re-addition of residues 63-77 with $\mathrm{N} 68 \mathrm{Q}+\mathrm{N} 72 \mathrm{Q}$ point mutations (chick TrkC LRRIg1 $1_{2 \mathrm{O}}$ ).

A series of mouse TrkC constructs (NCBI Ref. Seq. BC139764.1) were cloned into both pHLsec and pHL-Avitag3 vectors: LRR (amino acids 32-208), LRRIg1 (amino acids 32-302) and LRRIg2 (amino acids 32-398). A mouse TrkC TM (amino acids 32-463) construct was cloned into the pHLsec-monoVenus vector. The following mouse TrkC mutant constructs were generated by PCR: D240A + D242A (LRRIg1 and TM constructs), N68Q + N72Q (LRRIg1 $2 \mathrm{Q}$ and $\mathrm{TM}_{2 \mathrm{Q}}$ ) and removal of residues 63-77 (LRRIg1 $1_{\text {cryst }}$ ). Mouse NGL-3 (NCBI Ref. Seq. BC060263.1) ectodomain (NGL-3 Ecto; amino acids 1-574) and full-length (NGL-3 FL; amino acids 1-709) constructs were cloned into pHL-Avitag3 and pHLsec-monoCerulean vectors, respectively.

Protein purification and crystallization. For crystallization purposes, constructs were expressed in either HEK-293S GnTI ${ }^{-}$cells (chicken RPTP $\sigma$ Ig1-3, TrkC LRRIg $1_{\text {cryst }}$ and TrkC LRRIg1 $1_{2 \mathrm{O}}$ ) or HEK293-T cells treated with kifunensine (human RPTP $\sigma$ Ig1-FN3) following transient transfection using polyethylenimine ${ }^{51}$. The proteins were purified from $0.2-\mu \mathrm{m}$-filtered cell culture media by immobilized nickel affinity chromatography (Chelating Sepharose Fast Flow, GE Healthcare) followed by size-exclusion chromatography in $10 \mathrm{mM}$ HEPES, $150 \mathrm{mM} \mathrm{NaCl}, \mathrm{pH} 7.5$.

Crystallization trials, using $100 \mathrm{nl}$ protein solution plus $100 \mathrm{nl}$ reservoir solution in sitting-drop vapour diffusion format were set up in 96-well Greiner plates using a Cartesian Technologies robot, and plates were subsequently maintained at $20.5^{\circ} \mathrm{C}$ in a TAP Homebase storage vault. The crystallization conditions yielding diffraction quality crystals were: $10 \%$ polyethylene glycol (PEG) $20 \mathrm{k}, 20 \%$ PEG MME 550, $0.1 \mathrm{M}$ bicine/Tris $\mathrm{pH} 8.5,0.03 \mathrm{M}$ sodium nitrate, $0.03 \mathrm{M}$ disodium hydrogen phosphate, $0.03 \mathrm{M}$ ammonium sulphate (chicken RPTP $\sigma$ Ig1-2:TrkC LRRIg $1_{\text {cryst }}$ complex $2.5 \AA$ data set), 10\% w/v PEG MME $5 \mathrm{k}, 0.1 \mathrm{M}$ HEPES $\mathrm{pH} 7.0$, $5 \%$ w/v Tacsimate (chicken RPTP $\sigma$ Ig1-3:TrkC LRRIg $1_{2 Q}$ complex $3.05 \AA$ data set) and $10 \%$ PEG $400,0.01 \mathrm{M}$ magnesium chloride, $0.1 \mathrm{M}$ potassium chloride, $0.05 \mathrm{M}$ MES pH 6.0 (human RPTP $\sigma$ Ig1-FN3).

Data collection and processing. Crystals were cryoprotected using a 25-30\% solution of ethylene glycol (chicken RPTP $\sigma$ Ig1-2:TrkC LRRIg $1_{\text {cryst }}$ and RPTP $\sigma$ Ig1-3:TrkC LRRIg1 ${ }_{2 Q}$ complexes) or $25 \%$ propylene glycol (human RPTP $\sigma$ Ig1FN3) and then flash-cooled at $100 \mathrm{~K}$. X-ray diffraction data were collected at the I03 (chicken RPTP $\sigma$ Ig1-3:TrkC LRRIg1 2 Q ; wavelength $0.9763 \AA$ ) and I04 (chicken RPTP $\sigma$ Ig1-2:TrkC LRRIg1 $1_{\text {cryst }}$; wavelength $0.9200 \AA$ ) beamlines Diamond Light Source, Oxfordshire, UK and the ID29 (human RPTP $\sigma$ Ig1-FN3; wavelength $0.9788 \AA$ ) beamline at the European Synchotron Radiation Facility, Grenoble, France. The diffraction images were indexed, integrated, scaled and merged using the xia2 data-processing suite ${ }^{52}$. We used $R_{\text {pim }}, I / \sigma I$ and $\mathrm{CC}_{1 / 2}$ (ref. 53) statistics in the highest-resolution shell (with criteria $R_{\mathrm{pim}}<100 \%, I / \sigma I>1.5$ and $\mathrm{CC}_{1 / 2}>50 \%$ ) to determine our high-resolution cutoffs (see Table 1).

Molecular replacement was used to phase all three crystal structures, using human and chicken RPTP $\sigma$ Ig1-2 (PDB ID: 2YD3 and 2YD4), human RPTP $\sigma$ Ig3 (from PDB ID: 2YD9), a C $\alpha$ model for human RPTP LAR FN4 (PDB ID: 2DJU) and models of the chicken TrkC LRR and first Ig domain (Ig1) generated using the SWISS-MODEL web interface ${ }^{54}$, as search models in Phaser ${ }^{55}$. Manual model adjustment was performed in $\operatorname{Coot}^{56}$ and the Refmac ${ }^{57}$, Phenix ${ }^{58}$ and Buster (Global Phasing Ltd) ${ }^{59}$ suites used for refinement (applying translation libration screw-motion restraints for all structures and local non-crystallographic symmetry restraints for RPTP $\sigma: T r k C$ complex structures). Stereochemical properties of all models were accessed using MolProbity ${ }^{60}$. Ramachandran statistics: human RPTP $\sigma$ Ig1-FN3, 94.0\% most favoured, 6.0\% additionally allowed and no disallowed rotamers; chicken RPTP $\sigma$ Ig1-2:TrkC LRRIg1 $1_{\text {cryst }}$, $98 \%$ most favoured, $2 \%$ additionally allowed and no disallowed rotamers; chicken RPTP $\sigma$ Ig1-3:TrkC LRRIg $1_{2 \mathrm{Q}}, 96.6 \%$ most favoured, $3.2 \%$ additionally allowed and $0.2 \%$ disallowed rotamers. Full data collection and refinement statistics are given in Table 1.

The 3.15-Å human RPTP $\sigma$ Ig1-FN3 crystal structure contains one molecule per asymmetric unit; amino residues $35-601$, an additional C-terminal G residue derived from the expression vector and two GlcNAc residues at $\mathrm{N}$-linked glycosylation sites N250 and N295.

The $2.5 \AA$ chicken RPTP $\sigma$ Ig1-2:TrkC LRRIg1 $1_{\text {cryst }}$ crystal structure contains amino-acid residues 32-302 for two molecules, A and B, of TrkC LRRIg1 (lacking residues 63-77 absent in the TrkC LRRIg1 cryst expression construct and residues 258-261 on a disordered Ig1 loop, but with ETG (A) and G (B) additional N-term residues and GT (A) and G (B) additional C-term residues derived from the expression plasmid) and residues 29-227, 29-226 and 29-228 for three molecules, C, D and E, of RPTP $\sigma$ Ig1-2 (with the exception of a disordered loop for each molecule, 68-71(C), 68-70(D) and 68-73(E)). Five TrkC $N$-linked glycosylation sites were modelled; initial $\mathrm{N}$-acetylglucosamine (GlcNAc) residues, covalently bonded to N79 (A and B), N203 (A) and N272 (A and B) were included in the structure. Inspection of the crystal packing clearly demonstrates that the Ig3 domains of the RPTP $\sigma \operatorname{Ig} 1-3$ crystallization protein are not present in these crystals. Proteolytic cleavage is the most likely reason for their absence, as we have commonly seen cleavage of wild-type RPTP $\sigma$ Ig1-3 at the Ig2-Ig3 linker during crystallization trials. The previously reported human RPTP $\sigma$ Ig1-2 crystal structure was obtained via proteolysis of Ig1-3 during crystallization (PDB ID 2YD3). To produce crystals of intact human RPTP $\sigma$ Ig1-3, R227Q + R228N (residue numbering relative to chicken RPTP $\sigma$ ) point mutations were previously introduced to disrupt a potential furin protease motif ${ }^{14}$. However, RPTP $\sigma 227$ and R228 are required for full RPTP $\sigma: T r k C$ binding (Fig. 4c; Supplementary Fig. 8e,f; Supplementary Table 1) and therefore the RPTP $\sigma$ Ig1-3 R227Q + R228N protein was not used in crystallization trials in this study.

The $3.05 \AA$ chicken RPTP $\sigma$ Ig1-3:TrkC LRRIg $1_{2 \mathrm{Q}}$ crystal structure contains amino-acid residues 32-302 for three molecules, $\mathrm{A}, \mathrm{B}$ and $\mathrm{C}$, of TrkC LRRIg1 (lacking residues 59-68 (A), 59-68 (B) and 57-73 (C), but with TG (A), G (B) and $\mathrm{G}(\mathrm{C})$ additional $\mathrm{N}$-term residues derived from the expression plasmid) and residues 29-226, 29-227 and 29-225 for three molecules, D, E and F, of RPTP $\sigma$ Ig1-3 (with the exception of residues 67-71 in the disordered 'Lys-loop' for each molecule, for which density was visible, but a single conformation could not be built). Electron density was visible to indicate the presence of $\mathrm{N}$-linked glycosylation adjacent to N79 in TrkC molecules A-C, but only in molecule A could an initial GlcNAc residue be successfully refined. There was also evidence that N218 (electron density corresponding to molecule B) and N272 (electron density corresponding to molecule A) are also $N$-linked glycosylation sites. Sparse electron density is visible for RPTP $\sigma$ Ig3, suggesting disorder/multiple conformations of this domain.

The assignment of secondary-structure elements was performed using ksdssp ${ }^{61}$ The superposition of atomic models to compare the domain architecture between different structures was performed using $\mathrm{SHP}^{62}$, based on $\mathrm{C} \alpha$ positions. Crystallographic figures were created using PyMOL (Schrödinger, LLC) and $\mathrm{APBS}^{63}$ was used to calculate the electrostatic potential of solvent-accessible surfaces.

Surface Plasmon Resonance. All SPR experiments were performed on BIAcore T100 or T200 instruments. Ligand constructs were expressed in HEK293-T cells with no kifunensine treatment and purified via Ni-affinity chromatography. All contained a C-terminal BirA recognition site (Avitag) and were biotinylated enzymatically prior to immobilization to the surface of CM5 sensor chips (BIAcore) pre-coated with streptavidin (Sigma) using the BIAcore amine-coupling kit. Analyte constructs were expressed in either $\mathrm{GnTI}^{-}$HEK293-S or HEK293-T cells and purified as for crystallization purposes, described above.

Unless otherwise stated, experiments were all performed in $10 \mathrm{mM}$ HEPES $\mathrm{pH}$ $7.5,150 \mathrm{mM} \mathrm{NaCl}, 0.01 \%$ Tween 20 , at a temperature of $25^{\circ} \mathrm{C}$ and a flowrate of $20 \mu \mathrm{min}^{-1}$. Typically, each analyte sample was injected across the chip surfaces for $120 \mathrm{~s}$ and then a $300 \mathrm{~s}$ dissociation time was included to allow the signal to return to baseline. For equilibrium SPR experiments, serial two- or threefold dilutions of protein analyte were sequentially injected and all injection series were repeated in duplicate. No regeneration of the chip surfaces was required between analyte injections, except when measuring the interaction between chicken TrkC $\operatorname{LRRIg}_{2 \mathrm{Q}}$ and chicken RPTP $\sigma \operatorname{Ig} 1-3$, when a 30 s injection of $1 \mathrm{M} \mathrm{MgCl}_{2}$ was sufficient for the signal to return to baseline.

Scrubber2 (BioLogic Software) and Prism (GraphPad Software) were used for data analysis assuming the Langmuir model and a 1:1 ligand to analyte ratio. The signal for experimental flow cells was corrected by initial subtraction of a blank (only buffer injected as analyte across the flow cell of interest), followed by the subtraction of the reference signal from a mock-coupled flow cell (streptavidin, but no ligand bound). To estimate half-maximal inhibitory concentration values for heparan sulphate or heparin-dp10 inhibition of RPTP $\sigma: T r k C$ binding, nonlinear regression in Prism (GraphPad Software) was used to fit a variable-slope doseresponse curve to the experimental data, fixing top and bottom values according to control measurements (top; response units when no inhibitor present and bottom; response units when no protein analyte or inhibitor present), while the Hill Slope coefficient and half-maximal inhibitory concentration variables were left unrestrained.

Multi-Angle Light Scattering. MALS experiments were carried out on a Wyatt MALS/AFFFF System (Wyatt Technologies). Human RPTP $\sigma$ Ig1-FN3 and mouse TrkC LRRIg1 proteins were expressed in $\mathrm{GnTI}^{-}$HEK-293S cells and purified as described above. Size-exclusion chromatography was performed in $10 \mathrm{mM}$ Tris, $50 \mathrm{mM} \mathrm{NaCl}$, pH 7.5 on a Superdex200 HR10/30 column (GE Healthcare), attached to an Agilent chromatography system. An Optilab rEX Refractive Index detector and a Dawn Helios II Multi-Angle Light Scattering detector recorded the refractive index and light scattering of the samples upon elution from the sizeexclusion column. The Wyatt software ASTRA was used to analyse all the data collected.

Negative-stain EM. Human RPTP $\sigma$ Ig1-FN3 and sEcto proteins were negatively stained with $0.7 \%$ uranyl formate ${ }^{64}$. Images were recorded using an FEI electron microscope equipped with $\mathrm{LaB}_{6}$ filament operated at an acceleration voltage of $200 \mathrm{keV}$ at a magnification of $\times 55,000$ and a defocus value of approximately 
$-1.5 \mu \mathrm{m}$. All images were recorded using SO-163 film and developed with a Kodak D-19 developer at full strength for $12 \mathrm{~min}$ at $20^{\circ} \mathrm{C}$. The electron micrographs were digitized with a CoolScan 9000 (Nikon) using a step size $6.35 \mu \mathrm{m}$, and the pixels were binned by a factor of 3 . As a result, the specimen level pixel size was at $3.8 \AA$. To generate projection averages, particles were interactively selected using the WEB display program in SPIDER ${ }^{65}$ and windowed into $90 \times 90$ pixel (human RPTP $\sigma$ Igl-FN3) and $100 \times 100$-pixel (human RPTP $\sigma$ sEcto) images. Class averages were calculated using these windowed images over 10 cycles of K-means classification and multi-reference alignment specifying 150 classes $^{64}$.

SAXS. RPTP $\sigma$ Ig1-FN3 and sEcto proteins were deglycosylated by Endo-F1 and purified by SEC immediately prior to data collection. Solution scattering data were collected at beamline BM29 of the European Synchotron Radiation Facility ${ }^{66}$ at $293 \mathrm{~K}$ within a momentum transfer range of $0.01 \AA^{-1}<q<0.45 \AA^{-1}$, where $q=4 \pi \sin (\theta) / \lambda$ and $2 \theta$ is the scattering angle. X-ray wavelength was $0.995 \AA$ and data were collected on a Pilatus $1 \mathrm{M}$ detector. RPTP $\sigma$ Ig1-FN3 was measured at 1.33 and $5.33 \mathrm{gl}^{-1}$ and RPTP $\sigma$ sEcto at 1.00 and $4.00 \mathrm{gl}^{-1}$. Data reduction and calculation of invariants was carried out using standard protocols implemented in the ATSAS software suite ${ }^{67}$. A merged data set was obtained by merging the lowangle part of the low-concentration data set with the high-angle part of the highconcentration data set.

A pool of 10,000 independent model conformers was constructed using the program RANCH ${ }^{67}$ for both RPTP $\sigma$ Ig1-FN3 and sEcto by treating individual domains as beads-on-a-string. For RPTP $\sigma$ sEcto, a homology model for domains 8 and 9 was created using SWISS-MODEL ${ }^{54}$. Both pools contained conformer shapes ranging from collapsed or U-shaped to fully extended, as evidenced by their Gaussian $R_{\mathrm{G}}$ and $D_{\mathrm{MAX}}$ distributions. Ensemble selection using the experimental SAXS data as constraint with the programs GAJOE ${ }^{67}$ or $\mathrm{MES}^{68}$ indicated predominantly extended conformers, consistent with the experimentally determined $R_{\mathrm{G}}$ and $D_{\mathrm{MAX}}$. The MES-selected models were used as starting structures for further modelling. Missing loops and $\mathrm{N}$ - and $\mathrm{C}$ termini were added in extended conformations using the program Modeller. All-atom simulations of RPTP $\sigma$ Ig1-FN3 and sEcto was performed using the program AllosMod. For each starting structure, 30 independent pools of 100 models were generated. For the combined pool, calculation and fitting of scattering patterns was performed using the program FoXS, and automated selection of the minimal set of models satisfying the scattering data was performed using the program MES; this whole procedure was automated using the AllosMod-FoXS web server ${ }^{68}$.

Neuron-COS cell co-culture assays. Dissociated primary hippocampal neuron cultures were prepared from embryonic day 18 rat embryos ${ }^{69}$. For co-culture assays, COS- 7 cells were transfected and $24 \mathrm{~h}$ later were seeded onto neurons at 14 days in vitro ${ }^{70}$. As indicated, co-culture coverslips were incubated with $0.2 \mathrm{U} \mathrm{ml}^{-1}$ heparinase I, II and III or with $30 \mu \mathrm{g} \mathrm{ml}^{-1}(\sim 10 \mu \mathrm{M})$ heparin-dp10 (Iduron Ltd, UK) in glial conditioned medium. Co-cultures were fixed with $4 \%$ formaldehyde and $4 \%$ sucrose in phosphate-buffered saline (PBS) (pH 7.4) followed by permeabilization with $0.2 \%$ Triton X-100. For cell surface staining of TrkC, COS-7 cells were fixed without permeabilization. Fixed cultures were blocked in $3 \%$ bovine serum albumin and $5 \%$ normal goat serum in PBS for $30 \mathrm{~min}$ at $37^{\circ} \mathrm{C}$, and primary antibodies (overnight incubation at $4{ }^{\circ} \mathrm{C}$ ) then secondary antibodies $\left(1 \mathrm{~h}\right.$ at $37^{\circ} \mathrm{C}$ ) were applied in $3 \%$ bovine serum albumin and 5\% normal goat serum in PBS. Coverslips were mounted in elvanol (Tris- $\mathrm{HCl}$, glycerol and polyvinyl alcohol, with $2 \%$ 1,4-diazabicyclo[2,2,2] octane). The primary antibodies were: anti-TrkC (1:500; C44H5; Cell Signaling), anti-synapsin I (rabbit, 1:2,000; Millipore; AB1543P) for presynaptic terminals, anti-MAP2 (chicken polyclonal IgY; 1:2,000; Abcam; ab5392) for dendrites and anti-dephospho-tau (mouse mIgG2a 1:2,000, clone PC1C6; Millipore; MAB3420) for axons.

All image acquisitions, analyses and quantifications were performed by investigators blind to the experimental condition. For co-cultures, fields for imaging were chosen only by the Cyan Fluorescent Protein (CFP) or mVenus and phase-contrast channels, for the presence of CFP or mVenus-positive COS-7 cells in a neurite-rich region. The synapsin channel was thresholded and the total intensity of puncta within all regions positive for both CFP or mVenus and dephospho-tau but negative for MAP2 was measured. Analysis was performed using Fiji (ImageJ2), and GraphPad Prism 5. All data are reported as mean \pm s.e.m.

\section{References}

1. Siddiqui, T. J. \& Craig, A. M. Synaptic organizing complexes. Curr. Opin. Neurobiol. 21, 132-143 (2011).

2. Shen, K. \& Scheiffele, P. Genetics and cell biology of building specific synaptic connectivity. Annu. Rev. Neurosci. 33, 473-507 (2010).

3. Good, M. C., Zalatan, J. G. \& Lim, W. A. Scaffold proteins: hubs for controlling the flow of cellular information. Science 332, 680-686 (2011).

4. Craig, A. M. \& Kang, Y. Neurexin-neuroligin signaling in synapse development. Curr. Opin. Neurobiol. 17, 43-52 (2007).

5. Bell, C. H. et al. Structure of the repulsive guidance molecule (RGM)-neogenin signaling hub. Science 341, 77-80 (2013).

6. Johnson, K. G. \& Van Vactor, D. Receptor protein tyrosine phosphatases in nervous system development. Physiol. Rev. 83, 1-24 (2003).
7. Dunah, A. W. et al. LAR receptor protein tyrosine phosphatases in the development and maintenance of excitatory synapses. Nat. Neurosci. 8, 458-467 (2005).

8. Takahashi, H. \& Craig, A. Protein tyrosine phosphatases PTP $\delta, P T P \sigma$, and LAR: presynaptic hubs for synapse organization. Trends Neurosci. 36, 522-534 (2013).

9. Um, J. W. \& Ko, J. LAR-RPTPs: synaptic adhesion molecules that shape synapse development. Trends Cell Biol. 23, 465-475 (2013).

10. Aricescu, A. R., McKinnell, I. W., Halfter, W. \& Stoker, A. W. Heparan sulfate proteoglycans are ligands for receptor protein tyrosine phosphatase $\sigma . \mathrm{Mol}$. Cell. Biol. 22, 1881-1892 (2002).

11. Fox, A. N. \& Zinn, K. The heparan sulfate proteoglycan syndecan is an in vivo ligand for the drosophila LAR receptor tyrosine phosphatase. Curr. Biol. 15, 1701-1711 (2005).

12. Johnson, K. G. et al. The HSPGs syndecan and dallylike bind the receptor phosphatase LAR and exert distinct effects on synaptic development. Neuron 49, 517-531 (2006).

13. Shen, Y. et al. PTPsigma is a receptor for chondroitin sulfate proteoglycan, an inhibitor of neural regeneration. Science 326, 592-596 (2009).

14. Coles, C. H. et al. Proteoglycan-specific molecular switch for RPTP $\sigma$ clustering and neuronal extension. Science 332, 484-488 (2011).

15. Fisher, D. et al. Leukocyte common antigen-related phosphatase is a functional receptor for chondroitin sulfate proteoglycan axon growth inhibitors. J. Neurosci. 31, 14051-14066 (2011).

16. Fry, E. J., Chagnon, M. J., López-Vales, R., Tremblay, M. L. \& David, S. Corticospinal tract regeneration after spinal cord injury in receptor protein tyrosine phosphatase sigma deficient mice. Glia 58, 423-433 (2010).

17. Takahashi, H. et al. Postsynaptic TrkC and presynaptic PTP $\sigma$ function as a bidirectional excitatory synaptic organizing complex. Neuron 69, 287-303 (2011).

18. Kwon, S. K., Woo, J., Kim, S. Y., Kim, H. \& Kim, E. Trans-synaptic adhesions between netrin-G ligand-3 (NGL-3) and receptor tyrosine phosphatases LAR, protein-tyrosine phosphatase delta (PTPdelta), and PTPsigma via specific domains regulate excitatory synapse formation. J. Biol. Chem. 285, 13966-13978 (2010).

19. Yoshida, T. et al. Interleukin-1 receptor accessory protein organizes neuronal synaptogenesis as a cell adhesion molecule. J. Neurosci. 32, 2588-2600 (2012).

20. Yim, Y. S. et al. Slitrks control excitatory and inhibitory synapse formation with LAR receptor protein tyrosine phosphatases. Proc. Natl Acad. Sci. USA 110, 4057-4062 (2013).

21. Pulido, R., Serra-Pagès, C., Tang, M. \& Streuli, M. The LAR/PTP delta/PTP sigma subfamily of transmembrane protein-tyrosine-phosphatases: multiple human LAR, PTP delta, and PTP sigma isoforms are expressed in a tissuespecific manner and associate with the LAR-interacting protein LIP.1. Proc. Natl Acad. Sci. USA 92, 11686-11690 (1995).

22. Biersmith, B. H., Hammel, M., Geisbrecht, E. R. \& Bouyain, S. The immunoglobulin-like domains 1 and 2 of the protein tyrosine phosphatase LAR adopt an unusual horseshoe-like conformation. J. Mol. Biol. 408, 616-627 (2011).

23. Comoletti, D. et al. The macromolecular architecture of extracellular domain of $\alpha$ NRXN1: domain organization, flexibility, and insights into trans-synaptic disposition. Structure 18, 1044-1053 (2010).

24. Brasch, J., Harrison, O. J., Honig, B. \& Shapiro, L. Thinking outside the cell: how cadherins drive adhesion. Trends Cell Biol. 22, 299-310 (2012).

25. Aricescu, A. R. et al. Structure of a tyrosine phosphatase adhesive interaction reveals a spacer-clamp mechanism. Science 317, 1217-1220 (2007).

26. Ozkan, E. et al. Extracellular architecture of the SYG-1/SYG-2 adhesion complex instructs synaptogenesis. Cell 156, 482-494 (2014).

27. Woo, J. et al. Trans-synaptic adhesion between NGL-3 and LAR regulates the formation of excitatory synapses. Nat. Neurosci. 12, 428-437 (2009).

28. Takahashi, H. et al. Selective control of inhibitory synapse development by Slitrk3-PTP $\delta$ trans-synaptic interaction. Nat. Neurosci. 15, 389-398 (2012).

29. Yoshida, T. et al. IL-1 receptor accessory protein-like 1 associated with mental retardation and autism mediates synapse formation by trans-synaptic interaction with protein tyrosine phosphatase $\delta$. J. Neurosci. 31, 13485-13499 (2011).

30. Valnegri, P. et al. The X-linked intellectual disability protein IL1RAPL1 regulates excitatory synapse formation by binding PTP $\delta$ and RhoGAP2. Hum. Mol. Genet. 20, 4797-4809 (2011).

31. Wang, H. et al. Expression of receptor protein tyrosine phosphatase- $\sigma$ $($ RPTP $-\sigma)$ in the nervous system of the developing and adult rat. J. Neurosci. Res. 41, 297-310 (1995).

32. Valenzuela, D. M. et al. Alternative forms of rat TrkC with different functional capabilities. Neuron 10, 963-974 (1993).

33. Lein, E. S. et al. Genome-wide atlas of gene expression in the adult mouse brain. Nature 445, 168-176 (2007).

34. Elchebly, M. et al. Neuroendocrine dysplasia in mice lacking protein tyrosine phosphatase $\sigma$. Nat. Genet. 21, 330-333 (1999). 
35. Wallace, M. J. et al. Neuronal defects and posterior pituitary hypoplasia in mice lacking the receptor tyrosine phosphatase PTP $\sigma$. Nat. Genet. 21, 334-338 (1999).

36. Tessarollo, L. et al. Targeted deletion of all isoforms of the trkC gene suggests the use of alternate receptors by its ligand neurotrophin-3 in neuronal development and implicates trkC in normal cardiogenesis. Proc. Natl Acad. Sci. USA 94, 14776-14781 (1997).

37. Klein, R. et al. Disruption of the neurotrophin-3 receptor gene trkC eliminates la muscle afferents and results in abnormal movements. Nature 368, 249-251 (1994).

38. Horn, K. E. et al. Receptor protein tyrosine phosphatase sigma regulates synapse structure, function and plasticity. J. Neurochem. 122, 147-161 (2012).

39. Chen, X., Yoshida, T., Sagara, H., Mikami, Y. \& Mishina, M. Protein tyrosine phosphatase sigma regulates the synapse number of zebrafish olfactory sensory neurons. J. Neurochem. 119, 532-543 (2011).

40. Razi, N. \& Varki, A. Masking and unmasking of the sialic acid-binding lectin activity of CD22 (Siglec-2) on B lymphocytes. Proc. Natl Acad. Sci. USA 95, 7469-7474 (1998).

41. Allen, N. et al. Astrocyte glypicans 4 and 6 promote formation of excitatory synapses via GluA1 AMPA receptors. Nature 486, 410-414 (2012).

42. Irie, F., Badie-Mahdavi, H. \& Yamaguchi, Y. Autism-like socio-communicative deficits and stereotypies in mice lacking heparan sulfate. Proc. Natl Acad. Sci. USA 109, 5052-5056 (2012)

43. Siddiqui, T. J. et al. An LRRTM4-HSPG complex mediates excitatory synapse development on dentate gyrus granule cells. Neuron 79, 680-695 (2013).

44. de Wit, J. et al. Unbiased discovery of glypican as a receptor for LRRTM4 in regulating excitatory synapse development. Neuron 79, 696-711 (2013).

45. Uemura, T. et al. Trans-synaptic interaction of GluRdelta2 and neurexin through Cbln1 mediates synapse formation in the cerebellum. Cell 141, 1068-1079 (2010)

46. Siddiqui, T. J., Pancaroglu, R., Kang, Y., Rooyakkers, A. \& Craig, A. M. LRRTMs and neuroligins bind neurexins with a differential code to cooperate in glutamate synapse development. J. Neurosci. 30, 7495-7506 (2010).

47. Wu, Y., Vendome, J., Shapiro, L., Ben-Shaul, A. \& Honig, B. Transforming binding affinities from three dimensions to two with application to cadherin clustering. Nature 475, 510-513 (2011).

48. Park, H. \& Poo, M. M. Neurotrophin regulation of neural circuit development and function. Nat. Rev. Neurosci. 14, 7-23 (2013).

49. Aicher, B., Lerch, M. M., Muller, T., Schilling, J. \& Ullrich, A. Cellular redistribution of protein tyrosine phosphatases LAR and PTPsigma by inducible proteolytic processing. J. Cell Biol. 138, 681-696 (1997).

50. Krueger, N. X. et al. Functions of the ectodomain and cytoplasmic tyrosine phosphatase domains of receptor protein tyrosine phosphatase Dlar in vivo. Mol. Cell. Biol. 23, 6909-6921 (2003).

51. Aricescu, A. R., Lu, W. \& Jones, E. Y. A time- and cost-efficient system for high-level protein production in mammalian cells. Acta Crystallogr. D Biol. Crystallogr. 62, 1243-1250 (2006)

52. Winter, G. xia2: an expert system for macromolecular crystallography data reduction. J. Appl. Crystallogr. 43, 186-190 (2010).

53. Karplus, P. A. \& Diederichs, K. Linking crystallographic model and data quality. Science 336, 1030-1033 (2012).

54. Arnold, K., Bordoli, L., Kopp, J. \& Schwede, T. The SWISS-MODEL workspace: a web-based environment for protein structure homology modelling. Bioinformatics 22, 195-201 (2006).

55. McCoy, A. J. et al. Phaser crystallographic software. J. Appl. Crystallogr. 40, 658-674 (2007)

56. Emsley, P. \& Cowtan, K. Coot: model-building tools for molecular graphics. Acta Crystallogr. D Biol. Crystallogr. 60, 2126-2132 (2004).

57. Murshudov, G. N., Vagin, A. A. \& Dodson, E. J. Refinement of macromolecular structures by the maximum-likelihood method. Acta Crystallogr. D Biol. Crystallogr. 53, 240-255 (1997).

58. Adams, P. D. et al. PHENIX: a comprehensive Python-based system for macromolecular structure solution. Acta Crystallogr. D Biol. Crystallogr. 66, 213-221 (2010)

59. Blanc, E. et al. Refinement of severely incomplete structures with maximum likelihood in BUSTER-TNT. Acta Crystallogr. D Biol. Crystallogr. 60, 2210-2221 (2004).

60. Davis, I. W. et al. MolProbity: all-atom contacts and structure validation for proteins and nucleic acids. Nucleic Acids Res. 35, W375-W383 (2007).

61. Kabsch, W. \& Sander, C. Dictionary of protein secondary structure: Pattern recognition of hydrogen-bonded and geometrical features. Biopolymers 22, 2577-2637 (1983)
62. Stuart, D. I., Levine, M., Muirhead, H. \& Stammers, D. K. Crystal structure of cat muscle pyruvate kinase at a resolution of 2.6 A. J. Mol. Biol. 134, 109-142 (1979).

63. Baker, N. A., Sept, D., Joseph, S., Holst, M. J. \& McCammon, J. A. Electrostatics of nanosystems: Application to microtubules and the ribosome. Proc. Natl Acad. Sci. USA 98, 10037-10041 (2001).

64. Ohi, M., Li, Y., Cheng, Y. \& Walz, T. Negative staining and image classification-powerful tools in modern electron microscopy. Biol. Proced. Online 6, 23-34 (2004).

65. Frank, J. et al. SPIDER and WEB: processing and visualization of images in 3D electron microscopy and related fields. J. Struct. Biol. 116, 190-199 (1996).

66. Pernot, P. et al. Upgraded ESRF BM29 beamline for SAXS on macromolecules in solution. J. Synchrotron. Radiat. 20, 660-664 (2013).

67. Petoukhov, M. V. et al. New developments in the ATSAS program package for small-angle scattering data analysis. J. Appl. Crystallogr. 45, 342-350 (2012).

68. Guttman, M., Weinkam, P., Sali, A. \& Lee, K. K. All-atom ensemble modeling to analyze small-angle X-ray scattering of glycosylated proteins. Structure 21, 321-331 (2013).

69. Kaech, S. \& Banker, G. Culturing hippocampal neurons. Nat. Protoc. 1, 2406-2415 (2006).

70. Graf, E. R., Zhang, X., Jin, S.-X., Linhoff, M. W. \& Craig, A. M. Neurexins Induce differentiation of GABA and glutamate postsynaptic specializations via neuroligins. Cell 119, 1013-1026 (2004).

\section{Acknowledgements}

Coordinates and structure factors are deposited in the Protein Data Bank with PDB IDs 4PBV, 4PBW and 4PBX. We thank K. Harlos, T. Walter, A. Clayton, G. Sutton, N. Shanks and D. Staunton for assistance, F. Alder and L. Ciani (UCL) for provision of hippocampal cells in preliminary studies. We also thank staff at the European Synchrotron Radiation Facility (beamlines ID29 and BM29) and Diamond Light Source (beamlines I03 and I04, proposal mx8423). This work was funded by the UK Medical Research Council (G0700232 and L009609 to A.R.A. and G9900061 to E.Y.J.), Cancer Research UK (A10976 to E.Y.J.) and USA National Institutes of Health (MH070860 to A.M.C. and R01HD061543 from NICHD to T.N.). The Wellcome Trust Centre for Human Genetics is supported by Wellcome Trust grant 090532/Z/09/Z. N.M. was the recipient of a Wellcome Trust D.Phil. studentship, P.Z. is the recipient of a Michael Smith Foundation for Health Research Postdoctoral Fellowship, C.H.C. is a Human Frontiers Science Program long-term Postdoctoral Fellow (LT000100/2013), J.E. is supported by EMBO and Marie-Curie long-term postdoctoral fellowships, A.M.C. is a Canada Research Chair and A.R.A. is a UK Medical Research Council Senior Fellow.

\section{Author contributions}

A.R.A., E.Y.J. and C.H.C. designed the project and all authors contributed to data analysis and preparation of the manuscript. W.L. transiently expressed all proteins. C.H.C and N.M. cloned and purified all constructs and performed SPR and crystallographic analyses for the RPTP $\sigma: T r k C$ complexes. C.H.C. solved the RPTP $\sigma$ Ig1-FN3 crystal structure. T.N. and C.H.C. performed the negative-stain electron microscopy and processed and analysed these data. N.M. and J.E. collected and analysed all SAXS data. A.W.S. carried out cellular assays. P.Z. performed COS-hippocampal cell culture experiments and analysed these data together with A.M.C.

\section{Additional information}

Supplementary Information accompanies this paper at http://www.nature.com/ naturecommunications

Competing financial interests: The authors declare no competing financial interests

Reprints and permission information is available online at http://npg.nature.com/ reprintsandpermissions/

How to cite this article: Coles, C. H. et al. Structural basis for extracellular cis and trans RPTP $\sigma$ signal competition in synaptogenesis. Nat. Commun. 5:5209 doi: $10.1038 /$ ncomms6209 (2014)

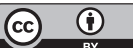

This work is licensed under a Creative Commons Attribution 4.0 International License. The images or other third party material in this article are included in the article's Creative Commons license, unless indicated otherwise in the credit line; if the material is not included under the Creative Commons license, users will need to obtain permission from the license holder to reproduce the material. To view a copy of this license, visit http://creativecommons.org/licenses/by/4.0/ 